\title{
The Co-expression Networks of differentially expressed RBPs with TFs and LncRNAs related to clinical TNM stages of Cancers
}

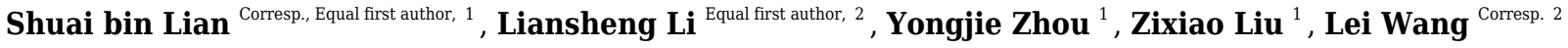 \\ ${ }^{1}$ College of Physics and Electronic Engineering, XinYang Normal University, Xinyang, HeNan, China \\ 2 College of Life Sciences, XinYang Normal University, Xinyang, HeNan, China \\ Corresponding Authors: Shuai bin Lian, Lei Wang \\ Email address: shuai_lian@xynu.edu.cn, wangleibio@126.com
}

Background: RNA-binding proteins (RBPs) play important roles in cellular homeostasis by regulating the expression of thousands of transcripts, which have been reported to be involved in human tumorigenesis. Despite previous reports of the dysregulation of RBPs in cancers, the degree of dysregulation of RBPs in cancers and the intrinsic relevance between dysregulated RBPs and clinical TNM information remains unknown. Furthermore, the co-expressed networks of dysregulated RBPs with transcriptional factors and IncRNAs also require further investigation. Results: Here, we firstly analyzed the deviations of expression levels of 1,542 RBPs from 20 cancer types and found that (1) RBPs are dysregulated in almost all 20 cancer types, especially in BLCA, COAD, READ, STAD, LUAD, LUSC and GBM with proportion of deviation larger than 300\% compared with non-RBPs in normal tissues. (2) Up- and down-regulated RBPs also show opposed patterns of differential expression in cancers and normal tissues. In addition, down-regulated RBPs show a greater degree of dysregulated expression than up-regulated RBPs do. Secondly, we analyzed the intrinsic relevance between dysregulated RBPs and clinical TNM information and found that (3) Clinical TNM information for two cancer types-CHOL and $\mathrm{KICH}$-is shown to be closely related to patterns of differentially expressed RBPS (DE RBPs) by co-expression cluster analysis. Thirdly, we identified ten key RBPs (seven downregulated and three up-regulated) in $\mathrm{CHOL}$ and seven key RBPs (five down-regulated and two up-regulated) in $\mathrm{KICH}$ by analyzing co-expression correlation networks. Fourthly, we constructed the co-expression networks of key RBPs between 1,570 TFs and 4,147 IncRNAs for $\mathrm{CHOL}$ and $\mathrm{KICH}$, respectively. Conclusions: These results may provide an insight into the understanding of the functions of RBPs in human carcinogenesis. Furthermore, key RBPs and the co-expressed networks offer useful information for potential prognostic biomarkers and therapeutic targets for patients with cancers at the $N$ and $M$ stages in two cancer types $\mathrm{CHOL}$ and $\mathrm{KICH}$. 


\section{The Co-expression Networks of differentially}

2 expressed RBPs with TFs and LncRNAs related to

3 clinical TNM stages of Cancers

4 Shuaibin Lian ${ }^{1 \text { }^{*}}$, Liansheng $\mathrm{Li}^{2 \#}$, Yongjie Zhou ${ }^{1}$, Zixiao Liu ${ }^{1}$, Lei Wang ${ }^{2 *}$

5

61 College of Physics and Electronic Engineering, Xinyang Normal University, Xinyang, Henan, China,

72 College of Life Sciences, Xinyang Normal University, Xinyang, Henan, China,

8

9 \# These two authors contribute equally.

10 Corresponding Author: Shuaibin Lian, shuai_lian@xynu.edu.cn; Lei Wang, wangleibio@126.com 


\section{The Co-expression Networks of differentially 15 expressed RBPs with TFs and LncRNAs related to 16 clinical TNM stages of Cancers}

1 College of Physics and Electronic Engineering, Xinyang Normal University, Xinyang, Henan, China, 21

\# These two authors contribute equally.

2 College of Life Sciences, Xinyang Normal University, Xinyang, Henan, China,

Corresponding Author: Shuaibin Lian, shuai_lian@xynu.edu.cn; Lei Wang, wangleibio@126.com

\section{ABSTRACT}

Background: RNA-binding proteins (RBPs) play important roles in cellular homeostasis by regulating the expression of thousands of transcripts, which have been reported to be involved in human tumorigenesis. Despite previous reports of the dysregulation of RBPs in cancers, the degree of dysregulation of RBPs in cancers and the intrinsic relevance between dysregulated RBPs and clinical TNM information remains unknown. Furthermore, the co-expressed networks of dysregulated RBPs with transcriptional factors and lncRNAs also require further investigation.

Results: Here, we firstly analyzed the deviations of expression levels of 1,542 RBPs from 20 cancer types and found that (1) RBPs are dysregulated in almost all 20 cancer types, especially in BLCA, COAD, READ, STAD, LUAD, LUSC and GBM with proportion of deviation larger than 300\% compared with non-RBPs in normal tissues. (2) Up- and down-regulated RBPs also show opposed patterns of differential expression in cancers and normal tissues. In addition, down-regulated RBPs show a greater degree of dysregulated expression than up-regulated RBPs do. Secondly, we analyzed the intrinsic relevance between dysregulated RBPs and clinical TNM information and found that (3) Clinical TNM information for two cancer types - $\mathrm{CHOL}$ and $\mathrm{KICH}$ - is shown to be closely related to patterns of differentially expressed RBPs (DE RBPs) by co-expression cluster analysis. Thirdly, we identified ten key RBPs (seven down-regulated and three up-regulated) in CHOL and seven key RBPs (five down- 
43 constructed the co-expression networks of key RBPs between 1,570 TFs and 4,147 lncRNAs for CHOL 44 and $\mathrm{KICH}$, respectively.

45 Conclusions: These results may provide an insight into the understanding of the functions of RBPs 46 in human carcinogenesis. Furthermore, key RBPs and the co-expressed networks offer useful information

47 for potential prognostic biomarkers and therapeutic targets for patients with cancers at the $N$ and $M$ stages 48 in two cancer types $\mathrm{CHOL}$ and $\mathrm{KICH}$.

\section{INTRODUCTION}

51

52

Recent research has highlighted the importance of changes in RNA metabolism in the mechanisms of carcinogenesis, including long non-coding RNAs (lncRNAs). RNA transcription, maturation, transportation, stabilization, degradation, and translation are molecular processes that regulate the cell cycle, as well as cell survival. The keys to regulating RNA metabolism are a group of proteins called RNA-binding proteins (RBPs), which participate in many steps at the post-transcriptional regulation level, and thereby determine the fate and function of each transcriptional transcript in the cell (Fu \& Ares, 2014; Stefanie, Markus \& Thomas, 2014; Moore \& Proudfoot, 2009). Furthermore, dysregulated expression of some RBPs can lead to disease, including neurological disorders and cancers (Wang et al., 2018; Kechavarzi and Janga, 2014). For instance, PAIP1 is proposed as a novel prognostic biomarker by affecting breast cancer cell growth (Piao et al., 2018). TRNAU1AP has been confirmed to play an important role in the regulation of cell proliferation and migration via the PI3K/Akt signaling pathway (Hu et al., 2018). SRPR is reported to regulate keratinocyte proliferation by affecting cell cycle progression and tend to show high expression in epidermal keratinocytes (Kim et al., 2016). RBMS3 has been found to inhibit breast cancer cell proliferation and tumorigenesis by inactivating the $\mathrm{Wnt} / \beta$-catenin signaling pathway (Yang, Quan \& Ling, 2018). In addition, overexpression of RPL34 is suggested to promote malignant proliferation of non-small cell lung cancer (NSCLC) (Yang et al., 2016). Silencing RPL34 plays a blocking role in cell proliferation and metastasis, but promoting cell apoptosis of oral squamous cell carcinomas (OSCCs) (Dai \& Wei, 2017; Liu et al., 2015). Moreover, the splicing regulator $P T B P 2$ is suggested to control a network of genes involved in germ cell adhesion, migration, and polarity and is also very essential for neuronal maturation (Qin et al., 2014; Molly, Leah \& Donny, 2017 \& Leah et al., 2015). However, some RBPs can act as tumor suppressors. For example, ZNFX1-AS1 is reported to suppress HCC progression via regulating the methylation of miR-9 (Wang et al., 2016). In addition, the silencing of SRSF7 affects the expression of osteopontin splice variants and decreases the proliferation rate of renal cancer cells (Boguslawska et al., 2016). Finally, RPL22/eL22, as a cancer-mutated RBP, tend to be anti-cancer via regulation of the MDM2-p53 feedback loop (Cao et al., 2017). 
Consequently, improving our understanding of the characteristics of RBPs and non-RNA-binding proteins (non-RBPs) is an essential step for understanding their roles in tumorigenesis. Even though recent studies have shown that RBPs are predominantly dysregulated in cancers relative to normal tissues (Wang et al., 2018; Bobak \& Sarath, 2014). But, the intensity of dysregulation of RBPs in different cancers is still need to be investigated. Furthermore, cancer is a complex genetic disease. The different developmental stages of cancer indicate different degrees of severity. The early detection of cancer is linked to improved survivorship. The tumor-node-metastases (TNM) system, formed by the Union for International Cancer Control (UICC) and the American Joint Committee on Cancer (AJCC), is the most widely used cancer staging system (Sorbin, Gospodarowicz \& Wittekind, 1992; American Joint Committee on Cancer, 2010). The TNM system provides information about the prognosis of the disease of patient based on pathologist evaluations of resected specimens. This information is also generally used to plan cancer treatment regimens. Due to the dysregulation of RBPs in many cancers, it is also interesting to determine whether there is an intrinsic relevance between RBP dysregulation and the developmental stages of cancers.

Transcription factors (TFs) perform the first step in interpreting the genome by recognizing specific DNA sequences to control transcription and gene expression (Lambert et al., 2018). As the unique gene class, TFs represent the proteins whose binding sites are affected by various regulatory variants in DNA. Accumulating Genome-wide association study (GWAS) shows that the mutations of TFs or TF-binding sites are closely related to many human cancers (reviewed in Deplancke et al., 2016), such as gastric cancer(Yin et al., 2017), liver cancer (Cao et al., 2017), prostate cancer, colorectal cancer(Saijo et al., 2016 ) and breast(Humphries et al., 2017) cancer. Even though, several studies have emerged to identified the regulatory mechanisms and interactions(Drissi et al., 2015; Zhang et al., 2019). Yet, in most cases, we still do not know how to interpret the regulatory interactions between RBPs and TFs. Furthermore, long non-coding RNAs (lncRNAs)-transcripts of greater than 200 nucleotides are of vital importance in transcriptional and post-transcriptional levels(Ishizuka et al., 2014). Several evidences have demonstrated that the expression of IncRNAs is closely related to human diseases(Yuan et al., 2017; Vergara et al., 2012), such as viral infections, neurological disease and cancers(Gibb, Brown \& Lam, 2011). Especially, the expression levels of lncRNAs in tumor tissues are significantly different compared with the normal tissues(Gloss and Dinger, 2015).Moreover, lncRNAs have merged as the novel biomarkers in several diseases diagnosis and targets for therapeutics(Zhou et al., 2013). Now, it is well established that transcription factors and long non-coding RNA have played a central role in the genetics of human diseases(Laurent et al., 2015). Yet, up to now, we are far from being able to know the regulatory interactions and mechanisms of RNA-binding proteins (RBPs) with TFs and lncRNAs. For example, whether there is an interaction between RBPs and TFs in the process of carcinomatosis and what types of 
110 TFs affect the regulatory interaction most? And, RBPs in which stage of cancers affect the expression of 111 lncRNA most?

112 To comprehensively characterize intensity of dysregulated RBPs from many human cancers (Wang 113 et al., 2018) and construct their interactive networks with TFs and lncRNAs related to TNM stages, we

114 first assessed the deviations of gene expression levels of RBPs and non-RBPs in 20 types of cancerous 115 and normal (control) tissues, respectively (Chang et al., 2013) and analyzed the biological and molecular 116 functions of dysregulated RBPs. Second, we analyzed the relationship between RBP dysregulation and 117 TNM system clinical data of 5,093 patients across 13 types of cancers. We found out two types of cancers 118 --cholangiocarcinoma (CHOL) and kidney chromophobe (KICH)--show a significant relationship 119 between RBP dysregulation and TNM stage information. Third, we constructed the interaction networks 120 for dysregulated RBPs related to TNM stage information and TFs of CHOL and KICH, respectively. 121 Fourth, we constructed the interaction networks for key RBPs related to metastases (M) stage and 122 lncRNAs for CHOL and KICH, respectively (see Fig. 1, which outlines the computational workflow, and 123 Materials and methods). This enabled us to identify the key regulatory RBPs for both CHOL and KICH 124 cancers.

\section{Materials \& Methods}

126 Differential expression analysis

127 We downloaded the original data including count matrix, expression FPKM values and clinical 128 information of 20 cancer types and paired normal tissues from TCGA data base using SangerBox tool 129 (SangerBox, http://sangerbox.com/). Then, we applied edgeR (Robinson et al., 2010) and DESeq2 130 (Anders S., 2009) to select differentially expressed genes (DEGs) from count matrix for each cancer type 131 with parameter padj $<0.05$, and $|\log 2 \mathrm{FC}|>1$. Thirdly, according 1,542 RBP genes information (Stefanie, 132 Markus \& Thomas, 2014), we divided the DEGs into two types for each cancer types: RBPs and non133 RBPs. The expression FPKM values were used to compute the degree of dysregulation of DEGs and 134 construct the co-expression networks. Clinical TNM information for 5,093 patients were used to co135 expressed gene module detection. Our datasets contained 1,542 RBPs, 1,570 TFs and 4,147 lncRNAs, 136 data acquisition information was presented in availability of data and materials section. The 'pheatmap' 137 package in $\mathrm{R}$ was used to generate a heatmap of differentially expressed RBPs shared by 16 cancer types 138 in six systems based on log2-fold change values. Pearson coefficient (R) was used to judge the similarity 139 of RBPs genes in different cancers occurring at related tissues have similar expression patterns. The gene 140 cluster with $\mathrm{R}>0.5$ was considered as having the similar expression pattern. Gene ontology categorization 141 analysis tool DAVID (Huang, Sherman \& Lempicki, 2009) was used to determine biological processes 142 and molecular functions of DE RBPs. 


\section{Standard deviation and proportion of dysregulation}

144

145

146

147

148

149

150

151

152

153

154

155

156

157

158

159

160

161

162

163

164

165

166

167

168

169

170

171

In order to investigate the degree of dysregulation of RBPs, we computed the standard deviation of differentially expressed genes listed in Table 1 using 'std' function in software 'MATLAB' version R2015b, respectively. In detail, we firstly computed the standard deviation of differentially expressed RBPs in 20 cancers and paired normal tissues (Fig. 2A). Similarly, we next computed the standard deviation of differentially expressed non-RBPs in 20 cancers and paired normal tissues (Fig. 2B).

The definition of the standard deviation is as follows:

$$
\sigma=\frac{1}{N} \sqrt{\sum_{i=1}^{N}\left(x_{i}-\mu\right)^{2}}
$$

Here, $N$ is the number of genes, $x_{i}$ is the expression FPKM value of the $i$ th gene, ${ }^{\mu}$ is the mean FPKM value of all $N$ genes. Larger $\sigma$ values represent gene expression values that deviate from the mean value to a greater degree, which is indicative of greater gene dysregulation. The standard deviation values for RBP expression data is presented in additional files (Table S1)

In order to more clearly see the expression differences of RBPs and non-RBPs in cancers and normal tissues, we further computed their relative deviations. In detail, we first computed the relative deviation of RBPs in cancers and paired normal tissues, and then we computed the relative deviation of non-RBPs in cancers and normal tissues (Fig. 2C). The relative proportion of deviation is computed using the

$$
p=\frac{\sigma_{c}}{\sigma}
$$

following function: $\quad \sigma_{n}$. Here, $\sigma_{c}$ and $\sigma_{n}$ are the standard deviations of the gene expressions in cancers and normal tissues respectively.

Similarly, in order to investigate the expression differences of up- and down-regulated RBPs, we also computed the standard deviation of them in cancers and normal tissues, respectively (Fig. 2(D,E)). Finally, the mean expression values of up- and down-regulated RBPs in 20 cancers and paired normal tissues were computed and presented in Fig. 2(F,G).

\section{Weighted co-expression network analysis}

Weighted gene co-expression network analysis (WGCNA) (Peter \& Steve, 2008) is a comprehensive $\mathrm{R}$ package that summarizes and standardizes methods and functions for co-expression network analysis. Module detection function of WGCNA was used to detect the correlations between co-expression gene modules and the clinical TNM information for 13 types of cancers with default settings one by one. And then, the threshold of correlation coefficient $R>0.5$ and statistical significance $P<0.05$ was used to select the cancer type whose TNM information related to co-expressed gene module. Genes with same 
172 expression pattern were clustered into one module and marked with same color. And then, the network

173 construction module was used to construct co-expression networks for RBPs with TFs and lncRNAs in

174 the CHOL and KICH cancer types, respectively. The detailed steps of constructing networks are as

175 follows. Firstly, WGCAN network construction tool was used to generate the nodes and edges of genes

176 by computing correlations of expression values. The nodes corresponded to genes, and the edges were

177 determined by the pairwise correlations between the expression levels of genes. The corresponding called

178 function in the R package was 'blockwiseModules' and the parameters were set as follows: 'powers $=10$, 179 minModuleSize $=30$, mergeCutHeight $=0.25$, other parameters we set to the default setting. Secondly, 180 nodes with correlation $r<0.5$ and edges with weighted threshold $<0.3$ were removed. Finally, Cytoscape 181 (https://cytoscape.org/) tool was used to plot the interactions using the nodes and edges of conserved 182 genes.

183 Clinical TNM information processing

184 Clinical TNM information for 5,093 patients exhibiting 13 types of cancers downloaded from the 185 TCGA database presented in additional files (Table S4). Generally, in the TNM system, ' $T$ ' refers to a 186 primary tumor, ' $T 1 \sim T 4$ ' represents the severity of primary cancer according to the increase in tumor 187 volume and the extent of involvement of adjacent tissues, and ' $T 0$ ' indicates no primary tumor. ' $\mathrm{N}$ ' 188 represents the tumor spreading to regional lymph nodes. 'N1 N3' represents the degree of spreading 189 according to the extent of lymph node involvement. ' $M$ ' refers to tumor metastasis. No distant metastasis 190 is expressed by ' $M O$ ', and distant metastasis is expressed by ' $M 1$ '. To investigate the correlation between 191 TNM information and gene expression values using WGCNA, we converted the TNM information into a 192 weighted matrix. For example, if TNM information for a patient was 'T1-N3-M1', the corresponding 193 weighted array is [ [ $\left.\begin{array}{lll}1 & 3 & 1\end{array}\right]$.

\section{Statistical methods}

We used the Mann-Whitney U-test (function 'ranksum' in software'MATLAB' version R2015b) to 196 examine whether there is statistical significance between given two samples, the default significance level is 0.05 (Lian et al., 2018).

The deviations of expression levels of RBPs between cancerous and normal tissues

To investigate the degrees of dysregulation of RBPs in many human cancers, we computed the standard deviations of gene expression levels of RBPs and non-RBPs in 20 types of cancerous and normal 
204

205

206

207

208

209

210

211

212

213

214

215

216

217

218

219

220

221

222

223

224

225

226

227

228

229

230

231

232

233

234

235

236

variation in expression in almost all types of cancer ( $\boldsymbol{P}<\mathbf{0 . 0 5}$, Mann-Whitney $\boldsymbol{U}$ test, Fig. 2A). In contrast, non-RBPs show considerably less variation in expression in cancers relative to normal tissues ( $\boldsymbol{P}<0.05$, Mann-Whitney $\boldsymbol{U}$ test, Fig. 2B). These results indicate that RBPs show a greater degree of dysregulated expression than non-RBPs in almost 20 types of cancers. Furthermore, the degrees of dysregulation of RBPs in different cancer types are significantly different. In particular, RBPs show severely dysregulated expression in eight types of cancers, including BLCA, LUAD, STAD, READ, LUSC, GBM and COAD. For these cancers, the corresponding relative proportions of dysregulation relative to normal tissues are $365.5 \%, 415.8 \%, 446.4 \%, 456.3 \%, 494.5 \%, 540.9 \%$ and $759.1 \%$, respectively (Fig. 2C, Table S1). Interestingly, up-regulated RBPs show a smaller standard deviation in expression in cancer tissue than in normal tissue $(\boldsymbol{P}<\mathbf{0 . 0 5}$, Mann-Whitney $\boldsymbol{U}$ test, Fig. 2D); However, down-regulated RBPs show considerable differences in the standard deviation of their expression in cancer tissue relative to normal tissues, especially in $\mathrm{KICH}$, KIRC, KIRP, COAD, LUSC, CESC, UCEC, and GBM ( $\boldsymbol{P}<\mathbf{0 . 0 5}$, Mann-Whitney $\boldsymbol{U}$ test, Fig. 2E). This may indicate that down-regulated RBPs are more dysregulated in cancers than are up-regulated RPBs. Finally, biological process enrichment analyzing indicates both up- and down-regulated RBPs were highly enriched in the processes, such as rRNA metabolism, nuclear-transcribed mRNA catabolism, and ncRNA processing (Additional files, Fig. S1). In addition, up-regulated RBPs were also enriched in mitochondrial gene expression and in the regulation of mRNA metabolic processes, while down-regulated RBPs were enriched in ribosome biogenesis and rRNA processing.

\section{Up- and down-regulated RBPs show opposite expression patterns in} cancer and normal tissue

To investigate cancer-specific differences in RBP expression, we analyzed the standard deviations and mean expression values of up-regulated and down-regulated RBPs in 20 types of cancers and in normal tissues (see methods and materials). Our results for all 20 cancer types suggests that, compared to normal tissues, up- and down-regulated RBPs show opposite patterns of expression in almost all cancers. What's more, down-regulated RBPs tend to show the larger expression deviations in cancers than upregulated RBPs ( $\boldsymbol{P}<\mathbf{0 . 0 5}$, Mann-Whitney $\boldsymbol{U}$ test, Fig. 2(D,F)). In particular, in almost all types of cancers, down-regulated RBPs show larger expression values than up-regulated RBPs. Furthermore, the expression deviations of up-regulated RBPs in cancers are lower than in normal tissues. However, the expression deviations of down-regulated RBPs are considerably greater in cancer tissue than in normal tissue; this is especially true for BLCA, GBM, HNSC, LUAD, LUSC, STAD, BRCA, KICH, READ, and UCSC $(\boldsymbol{P}<\mathbf{0 . 0 5}$, Mann-Whitney $\boldsymbol{U}$ test, Fig. 2(E,G)). These results suggest that down-regulated RBPs show a severer dysregulation in cancers than up-regulated RBPs. Furthermore, the expression pattern of 
237 up- and down-regulated RBPs in 20 types of cancers are the opposite of those found in normal tissues.

238 What's more, molecular functions enrichment analyzing indicates that both up- and down-regulated RBPs

239 showed enrichment in: catalytic activity acting on RNA, mRNA and mRNA 3'-UTR binding, nuclease

240 and ribonuclease activity, and single-stranded RNA binding. In addition, we found enrichment in

241 translation factor activity and RNA binding for up-regulated RBPs, and in catalytic activity acting on

242 tRNAs for down-regulated RBPs (Additional files, Fig. S1). In addition, we also were able to identify

243 which specific biological processes and functions were regulated by up- and down-regulated RBPs, which

244 may reveal how the normal processes of cells can be altered in a way that leads to cell carcinomatosis.

245 These results provide a new insight into understanding the roles of up- and down-regulated RBPs in the 246 process of cell carcinogenesis.

247 RBPs of different cancers in same system have a similar expression 248 profile

249 To gain clearer insight into RBP expression in cancerous tissues, we analyzed expression heatmaps 250 of 16 types of cancers, except four types of glandular cancer (PAAD, THCA, PRAD, and HNSC). 251 Because the number of DE RBPs in these four types of cancers are too small (Table 1). We divided DE 252 RBPs into six scale systems according to type (i.e. organization of canceration) and analyzed the 253 expression profiles of 801 DE RPBs shared by all 16 cancers by system. In each system, we divided 254 RBPs into different types according to their expression values and Pearson coefficient $R$. Gene clusters 255 with $R>0.5$ were considered as having the similar expression pattern, which was shown in heatmaps. This 256 was true for all cancers except two cancers of the nervous system. The corresponding gene lists of RBPs 257 relevant for each system are presented in additional files (Table S2).

258 In the respiratory and liver and gall systems (Fig. 3(A,B)), two pairs of cancers in same systems, 259 LUAD and LUSC, CHOL and LIHC, show very similar RBP expression profiles. Among these two 260 cancer systems, $\mathrm{C} 1$ and $\mathrm{C} 4$ genes showed the co-expression patterns of RBP for high expression and low 261 expression respectively $(R>0.5)$. The proportions of co-expressed RBPs in the respiratory and liver and 262 gall systems were $88.8 \%$ and $76.8 \%$, respectively. However, RBP expression in two cancers of the 263 nervous system (PCPG and GBM) showed opposite expression patterns (Fig. 3C). The proportions of 264 highly expressed RBPs in PCPG and GBM cancer tissue were $60.5 \%$ and $36.1 \%$, respectively. Highly 265 expressed RBPs in PCPG showed a lower degree of expression in GBM, while highly expressed RBPs in 266 GBM showed lower expression in PCPG. The proportion of RBPs showing opposing patterns of 267 expression in PCPG and GBM was 68.2\% $(R<0.5)$. In systems containing three types of cancers (i.e. the 268 alimentary and reproductive systems) (Fig. 3(D,E)), the proportion of co-expressed RBPs decreased 269 slightly, reaching $70.4 \%$ and $71.5 \%(R>0.5)$, respectively. In the urinary system, which was affected by 
270 four types of cancers, the proportion of co-expressed RBPs reached its minimum value of $35.3 \%$ (Fig.

271 3F). Of the cancers of the urinary system, two (KIRC and KIRP) showed the strongest degree of RBP co-

272 expression reaching 85.6\% $(R>0.5)$. Taken together, our results suggest that RBP expression in different

273 cancers in similar tissues have similar expression profiles.

\section{Co-expressed gene regulatory networks correlate with clinical TNM}

275 stage

276 Next, we investigated whether expression patterns are closely related to developmental stages of

277 different cancers and constructed the co-expression networks for DE RBPs and non-RBPs using module

278 detection and network construction tools of WGCNA (Peter \& Steve, 2008). Results showed that for two

279 types of cancers - CHOL and $\mathrm{KICH}$ - clinical TNM stage information was closely related to patterns of

280 gene expression (Fig. 4). The results for the other 11 cancer types didn't satisfied the threshold (Methods 281 and material, Additional files, Fig. S2-Fig. S7).

282 In terms of module detection, we identified three modules (orange, green and red) related to the 283 cancer metastasis stage ( $M$-stage), as well as two modules (royal blue and red modules) closely related to 284 the regional lymph node stage ( $N$-stage) for CHOL (Fig. 4A). The red module was consistently in both 285 the $\mathrm{M}$ and $\mathrm{N}$ stages. The gene list for each corresponding module is presented in additional files (Table 286 S3). Furthermore, we found 10 differentially expressed RBPs in these three modules. These included six 287 RBPs (ACO1, PPARGC1A, PUS7, KHDC1, ELAVL3, and BICC1) in the green module, two RBPs 288 (PANBP17 and HNRNPA1) in the royal blue module, and two RBPs (DCPS and C2orf15) in the red 289 module. For $\mathrm{KICH}$, we found that the royal blue module was most closely related to the $M$-stage, with a correlation coefficient of $0.96(P<4 \mathrm{e}-15)$, while the red and green modules were closely related to the $N$ stage (Fig. 4B). The gene list for each corresponding module is presented in additional files (Table S3). Furthermore, we found seven DE RBPs related to different developmental stages of cancer. These

293

294

295

296

297

298

299

300

301

302 included four RBPs (TNRC6A, MECP2, ZCCHC14, and POLR2F) in the green module and three RBPs (TDRD1, TDRD9, and CELF4) in the red module. The regulatory networks of CHOL (Fig. 4C)revealed that (1) in each sub-network, one RBP interacts with almost all non-RBPs, suggesting that these RBPs are key regulators for each module; (2) the RBPs in different sub-networks interact with each other, indicating that they work together to regulate the corresponding developmental stage of the cancer. (3) we found that most key RBPs are down-regulated, the proportion is $80 \%$. In addition, in the red module, two RBPs - including one down-regulated RBP (C2orf15) and one up-regulated RBP (CDPS) also play such a regulatory role. For the regulatory networks of $\mathrm{KICH}$, we also identified three sub-networks that corresponded to the $M$ - and $N$-stages (Fig. 4D). First, we found that RBPs interacted with almost all nonRBPs in each module, indicating that RBPs are key regulatory factors of the genes in these modules. 
303 Second, we identified seven key RBPs in the three modules, of which five were down-regulated and two

304 were up-regulated. In the green module, we identified four key RBPs-TNRC6A, MECP2, ZCCHC14, 305 and $P O L R 2 F$ - all of which were down-regulated. In the red module, we identified two key up-regulated

306 RBPs (TDRD1 and TDRD9) and one key down-regulated RBP, CELF4. These results suggest that 307 dysregulated RBPs play a key role in the regulation of the development of the CHOL and KICH $M$-stage, 308 which may provide a new perspective for potential prognostic biomarkers and therapeutic targets for 309 patients with cancers at $M$ stages in two cancer types CHOL and $\mathrm{KICH}$.

\section{The networks of key RBPs and IncRNAs for CHOL and KICH}

311

312

313

314

315

316

317

318

319

320

321

322

323

324

325

326

327

328

329

330

331

332

333

334

335

336

To infer the potential regulatory mechanisms of lncRNAs with key RBPs related to $M$ stages, we constructed the co-expression networks of key RBPs and differentially expressed (DE) lncRNAs for $\mathrm{CHOL}$ and KICH respectively and performed Gene Ontology and functional enrichment analyses.

Ten key RBPs and 2,943 DE lncRNAs were used to construct the co-expression network for CHOL. The resulting co-expression network consisted of 2 key RBPs (down-regulation C2orf15 and upregulation DCPS) and 75 lncRNAs, which were grouped into three clusters (Fig. 5A). There were 63 upregulated lncRNAs, and the proportion of up-regulated lncRNAs in three clusters was $86 \%, 75 \%, 83.3 \%$, which probably suggest that up-regulated lncRNAs have a greater interaction with RBPs in the process of metastasis of CHOL cells. Functional enrichment analyzing demonstrated that cluster 1 and cluster 3 had similar functions and mainly enriched in functional categories involved in gene silencing and negative regulation of translation, such as post transcriptional gene silencing, negative regulation of translation and cellular amide metabolic process, cellular response to dsRNA, miRNA metabolic process. Cluster 2 had some special functions and mainly enriched in such as positive regulation of mRNA catabolic process, cellular response to interleukin-1 and calcium ion, RNA destabilization.

Seven key RBPs and 1,204 DE lncRNAs were used to construct the co-expression network for $\mathrm{KICH}$. The resulting co-expression network consisting of 3 key RBPs (up-regulated TDRD1 and TDRD9, down-regulated CELF4) and 227 lncRNAs. There are 177 down-regulated lncRNAs in the network and were grouped into four clusters (Fig. 5B). The corresponding proportion of down-regulated lncRNAs was $46.7 \%, 65.3 \%, 75 \%$ and $95 \%$, which probably suggest that down-regulated lncRNAs play more important roles in interacting with key RBPs TDRD1, TDRD9 and CELF4 in the process of metastasis of KICH cells. Functional enrichment analysis demonstrated that cluster 1 shared by three key RBPs mainly enriched in dsRNA fragmentation and production of miRNAs involved in gene silencing by miRNA. Cluster 2 regulated by TDRD9 mainly enriched in endoribonuclease and exon-exon junction complex, cluster 3 regulated by TDRD1 mainly enriched in mRNA catabolic process and regulation of mRNA metabolic process, cluster 4 regulated by CELF4 mainly enriched in transporting of RNA, mRNA and nucleic acids. Besides, cluster 1 and cluster 2 have some similar functions, such as telomere maintenance,

Peer) reviewing PDF | (2019:05:37707:1:2:NEW 28 Jul 2019) 
337 histone mRNA and miRNA metabolic process, dosage compensation. Cluster 3 and cluster 4 have some 338 similar functions, such as regulation of RNA stability, RNA localization and regulation of mRNA 339 catabolic process.

340 These results provide a new insight into the understanding of the interactions of key RBPs with 341 lncRNAs in the metastasis stage ( $M$ stage) of cancer cells.

\section{2}

343 KICH

344

345

346

347

348

349

350

351

352

353

354

355

356

357

358

359

360

361

362

363

364

365

366

367

368

369

To investigate the interactions of RBPs and TFs, we constructed the co-expression networks of DEG RBPs and TFs for $\mathrm{CHOL}$ and $\mathrm{KICH}$, respectively. The key regulatory RBPs were those (ten for CHOL and seven for $\mathrm{KICH}$ ) detected in above section.

The co-expression network of CHOL revealed two important insights. First, we found the five largest transcription factor families, they are $\mathrm{C} 2 \mathrm{H} 2-\mathrm{ZF}$, Homeodomain, Nuclear receptor, bHLH and $b Z I P$, and the corresponding proportion is $37 \%, 17 \%, 9 \%, 9 \%$ and $9 \%$ (Fig. 6A, B), which interacted with almost all differentially expressed RBPs. This result indicates that these transcription factors tend to show a co-expression pattern with DEG RBPs, which further suggest that they play a major regulatory role in RBP post-regulatory levels for CHOL. Second,we also identified several special TFs related to up- or down-regulated RBPs for CHOL. For example, Grainyhead, MADF, HMG-Sox and SAND are specific transcription factors associated with down-regulated RBPs and GTF2I-like, Myb-SANT, MADS box and CENPB are specific transcription factors associated with up-regulated RBPs for CHOL. The coexpression network for $\mathrm{KICH}$ also revealed the following insights. First, we found that the four largest transcription factor families, they are $b H L H, b Z I P, C 2 H 2-Z F$ and Nuclear receptor, the proportion is $54 \%, 9 \%, 7 \%$ and 7\% (Fig. 6C, D). Notably, transcription factor $b H L H$ interact with all RBPs and it accounts for more than half of all interacted transcription factors, which indicate that $b H L H$ transcription factor probably involved in regulation of all differentially expressed RBPs for $\mathrm{KICH}$. Second, the proportion of up- and down-regulated RBPs co-expressed with TFs is $57 \%$ and $43 \%$ respectively. But, the up-regulated RBPs tend to show more interactions with TFs (Fig. 6C). These results provide insights into understanding the mechanism of interaction between transcription factors and RBPs.

\section{DISCUSSION}

RNA-binding proteins have been shown to be the key units to regulating RNA metabolism (Fu \& Ares, 2014; Stefanie, Markus \& Thomas, 2014; Moore \& Proudfoot, 2009) and dynamically interact with both coding and noncoding RNA (Kim et al., 2016). Furthermore, recent studies have shown that RBPs are down-regulated in cancers (Wang et al., 2018), but the study of 16 tissues from 80 healthy individuals indicated that RBPs show the higher expression than non-RBPs (Bobak \& Sarath, 2014). Consequently, 
370 we investigated the comprehensive expression differences of RBPs and non-RBPs simultaneously in

371 cancers and normal tissues. Results indicate that RBPs are significantly dysregulated in cancers. In

372 particular, recent studies have confirmed that RBPs show severely dysregulated expression in 373 BLCA(Kato et al., 2012), LUAD(Dong et al., 2018), STAD(Hapkova et al., 2013), READ, LUSC(Shi et 374 al., 2017), GBM(Pavlyukov et al., 2018) and $\operatorname{COAD}($ Saki et al., 2016). Furthermore, up- and down375 regulated RBPs tend to show opposite patterns of differential expression in cancers and normal tissues 376 (Fig. 2(D,E)). Up-regulated RBPs show higher expression in normal tissues than down-regulated RBPs, 377 which is consistent with the results in (Bobak \& Sarath, 2014), but down-regulated RBPs show the 378 significantly higher expression in cancers than up-regulated RBPs (Fig. 2(F,G)), which is consistent with 379 results in (Wang et al., 2018). These results probably suggest a mechanism of RBPs in the process of 380 carcinomatosis by which the up-regulated RBPs tend to show lower expression but down-regulated RBPs 381 tend to show higher expression. Carcinogenesis is probably caused by the combined actions of the low

382 383 384 385 386 387 388 389 390 391 392 393 394 395 396 397 398 399 400 401 expression of up-regulated RBPs and the high expression of down-regulated RBPs. This mechanism may be useful for understanding the roles of RPBs and the design of targeted drugs for cancer therapy.

What's more, we found 10 key regulated RBPs for CHOL (Seven down-regulated RBPs, HNRNPA1, PANBP17, PUS7, KHDC1, ELAVL3, BICC1 and C2orf15; Three up-regulated RBPs, ACO1 PPARGC1A and $D C P S$ ) and 7 key regulated RBPs for $\mathrm{KICH}$ (Six down-regulated RBPs, TNRC6A, MECP2, ZCCHC14, CELF4 and POLR2F; Two up-regulated RBPs, TDRD1 and TDRD9), respectively. Notably, recent studies have shown the importance of these key RBPs. For instance, multiple PPARGC1A transcripts are more abundant and CNS-specific in Parkinson's disease (PD) (Soyal et al., 2019). KHDC1A is highly expressed in oocytes and induces endoplasmic reticulum apoptosis (Cai et al., 2012). Elavl3 is closely related to neurodegenerative diseases and play an important role in maintaining the axonal homeostasis of neurons (Ogawa et al., 2018). HNRNPA1, regulated by miR-503 and miR-424, is associated with breast cancer cell proliferation (Otsuka, Yamamoto \& Ochiya, 2018). DCPS is very essential for acute myeloid leukemia cell survival by interacting with pre-mRNA (Yamauchi et al., 2018). CELF4 plays an important role in brain development, the haploinsufficiency of CELF4 is associated with autism disorders (Barone et al., 2017). POLR2F is significantly high expression in colorectal carcinomas (Antonacopoulou et al., 2008) and potential molecule in carcinogenesis. TDRD1 is over-expressed in majority of 131 primary prostate tumors patients (Xiao et al., 2016). In all, these results have demonstrated that the key RBPs have played the important roles in other types of cell carcinomatosis and provide a new perspective for potential prognostic biomarkers and therapeutic targets for patients with cancers at the $N$ and $M$ stages in two cancer types $\mathrm{CHOL}$ and $\mathrm{KICH}$.

\section{CONCLUSIONS}


403

404

405

406

407

408

409

410

411

412

413

414

415

416

417

418

419

420

421

422

423

424

425

426

427

428

429

430

431

432

433

434

435

In this study, we analyzed detailed differences in the expression of RBPs and non-RBPs across 20 types of cancers and constructed the co-expression networks of dysregulated RBPs with TFs and lncRNAs for CHOL and $\mathrm{KICH}$, respectively. Our results indicate that: (1) RBPs are dysregulated in almost all 20 cancer types compared with normal tissues, especially in BLCA, COAD, READ, STAD, LUAD, LUSC and GBM with proportion of deviation larger than 300\% compared with non-RBPs in normal tissues. (2) Up- and down-regulated RBPs also show opposed patterns of differential expression in cancers and normal tissues. In addition, down-regulated RBPs show a greater degree of dysregulated expression than up-regulated RBPs do. (3) Clinical TNM information for two cancer types-CHOL and $\mathrm{KICH}$ - is shown to be closely related to patterns of differentially expressed RBPs (DE RBPs). (4) We constructed the co-expression networks of key RBPs between 1,570 TFs and 4,147 lncRNAs for CHOL and $\mathrm{KICH}$, respectively. By analyzing these networks, we identified ten key RBPs (of which seven were down-regulated and three up-regulated) in CHOL and seven RBPs (of which five were down-regulated and two up-regulated) in KICH. These key RBPs - and especially down-regulated RBPs-likely play important roles in cell carcinomatosis. This study lays the foundation for further efforts to understand the roles played by RBPs in human carcinogenesis and provides a new insight into identifying the potential prognostic biomarkers and therapeutic targets for patients.

\section{ACKNOWLEDGEMENTS}

Authors thank three anonymous reviewers for their comments on the manuscript. The linguistic editing and proofreading provided by TopEdit LLC during the preparation of this manuscript are acknowledged.

\section{ADDITIONAL INFORMATION AND DECLARATIONS}

\section{Consent for publication}

All contributors to this study (i.e. the authors themselves) consent to the publication of these results.

\section{Availability of data and materials}

We downloaded RNA-seq data for paired samples of cancerous and normal tissues derived for 20 tumor types from TCGA data base using SangerBox tool (SangerBox, http://sangerbox.com/). This dataset included gene expression count matrix, FPKM values, and clinical TNM information. Our datasets contained 1,542 RBPs, 1,570 TFs, 4,147 lncRNAs and TNM information of 5,093 patients. Data for 1,542 RBP genes were downloaded from Stefanie et al (Stefanie, Markus \& Thomas, 2014). 4,147 differentially expressed lncRNAs data were downloaded from starBase v2.0 (Li et al., 2013) and 1,570 transcriptional factors (TFs) were downloaded from Lambert et al (Lambert et al., 2018). Clinical TNM information for seven cancer types was not available. Detailed clinical TNM information of the 5,093 patients of remaining 13 cancer types are presented in the additional files (Table S4).

\section{Authors' information}

PeerJ reviewing PDF | (2019:05:37707:1:2:NEW 28 Jul 2019) 
4361 College of Physics and Electronic Engineering, Xinyang Normal University, Xinyang, China.

4372 College of Life Sciences, Xinyang Normal University, Xinyang, China.

\section{ADDITIONAL FILES}

439 Table S1. The standard deviation values for up-and down-regulated RBPs and non-RBPs in cancers and 440 normal tissues.

441 Figure S1. Biological process and molecular function enrichment analysis of 801 differentially expressed 442 RBPs shared by 16 types of cancer.

443 Table S2. The gene lists of RBPs relevant for each system.

444 Figure S2. The co-expression modules of BLCA and BRCA correlated with clinical TNM stage 445 information.

446 Figure S3. The co-expression modules of CESC and COAD correlated with clinical TNM stage 447 information.

448 Figure S4. The co-expression modules of KIRC and KIRP correlated with clinical TNM stage 449 information.

450 Figure S5. The co-expression modules of LIHC and LUAD correlated with clinical TNM stage 451 information.

452 Figure S6. The co-expression modules of LUSC and READ correlated with clinical TNM stage 453 information.

454 Figure S7. The co-expression modules of STAD correlated with clinical TNM stage information.

455 Table S3. The gene list for each corresponding modules for CHOL and KICH.

456 Table S4. The clinical TNM information for 5,093 patients of 13 types of cancers.

\section{REFERENCES}

458 American Joint Committee on Cancer. Cancer staging manual. 7 edition. Chicago, IL: Springer; 2010. p649.

459

460

461

462

463

464

465

466

467

468
Anders, S. 2009. Differential gene expression analysis based on the negative binomial distribution. Journal of Marine Technology \& Environment 2(2).

Antonacopoulou AG, Grivas PD, Skarlas L, Kalofonos M, Scopa CD, Kalofonos HP. 2008. POLR2F, ATP6V0A1 and PRNP expression in colorectal cancer: new molecules with prognostic significance. Anticancer Res 28(2B):1221-1227. DOI 10.1245/s10434-007-9682-3.

Barone R, Fichera M, De Grandi M, Battaglia M, Lo Faro V, Mattina T, Rizzo R. 2017. Familial $18 \mathrm{q} 12.2$ deletion supports the role of RNA-binding protein CELF4 in autism spectrum disorders. Am J Med Genet A 173(6):1649-1655. DOI 10.1002/ajmg.a.38205.

Bobak Kechavarzi, Sarath Chandra Janga. 2014. Dissecting the expression landscape of RNA-binding proteins in human cancers. Genome Biology 15: R14, 1-16. DOI 10.1186/gb-2014-15-1-r14.

Peer] reviewing PDF | (2019:05:37707:1:2:NEW 28 Jul 2019) 
469

470

471

472

473

474

475

476

477

478

479

480

481

482

483

484

485

486

487

488

489

490

491

492

493

494

495

496

497

498

499

500

501

502

503

504

505

Boguslawska J, Sokol E, Rybicka B, Czubaty A, Rodzik K, Piekielko-Witkowska A. 2016. MicroRNAs target SRSF7 splicing factor to modulate the expression of osteopontin splice variants in renal cancer cells. Gene Dec 31, 595(2):142-149. DOI 10.1016/j.gene.2016.09.031.

Cai C, Liu J, Wang C, Shen J. 2012. KHDC1A, a novel translational repressor, induces endoplasmic reticulumdependent apoptosis. DNA Cell Biol Sep, 31(9):1447-57. DOI 10.1089/dna.2012.1682.

Cao B, Fang Z, Liao P, Zhou X, Xiong J, Zeng S, Lu H. 2017. Cancer-mutated ribosome protein L22 (RPL22/eL22) suppresses cancer cell survival by blocking p53-MDM2 circuit. Oncotarget Oct 6, 8(53):9065190661. DOI 10.18632/oncotarget.21544.

Castello A, Fischer B, Hentze MW, Preiss T. 2013. RNA-binding proteins in Mendelian disease. Trends Genet 29:318-327. DOI 10.1016/j.tig.2013.01.004.

Chang K, Creighton C J, Davis C, Donehower L, Drummond J, Wheeler D. 2013. The Cancer Genome Atlas Pan-Cancer analysis project. Nature Genetics, 45(10):1113-1120. DOI 10.1038/ng.2764.

Dai J, Wei W. 2017. Influence of the RPL34 gene on the growth and metastasis of oral squamous cell carcinoma cells. Arch Oral Biol 83:40-46. DOI 10.1016/j.archoralbio.2017.06.035.

Deplancke Bart, Alpern Daniel, Gardeux Vincent. 2016. The Genetics of Transcription Factor DNA Binding Variation. Cell, 166(3). DOI 10.1016/j.cell.2016.07.012.

Drissi Romain, Dubois Marie-Line, Douziech Mélanie, Boisvert François-Michel. 2015. Quantitative Proteomics Reveals Dynamic Interactions of the Minichromosome Maintenance Complex (MCM) in the Cellular Response to Etoposide Induced DNA Damage. Molecular \& cellular proteomics: MCP, 14(7):20022013. DOI 10.1074/mcp.M115.048991.

Fei Dong, Cen Li, Pu Wang, Xiaoya Deng, Qinli Luo, Xiaokui Tang, Li Xu. 2018. The RNA binding protein tristetraprolin down-regulates autophagy in lung adenocarcinoma cells. Experimental Cell Research, 367(1). DOI 10.1016/j.yexcr.2018.03.028.

Fu, X D, and Ares M. 2014. Context-dependent control of alternative splicing by RNA-binding proteins. Nat. Rev. Genet 15, 689-701. DOI 10.1038/nrg3778.

Gibb E A, Brown C J, Lam W L. 2011. The functional role of long non-coding RNA in human carcinomas. Molecular Cancer, 10(1):38. DOI 10.1186/1476-4598-10-38.

Gloss B S, Dinger M E. 2015. The specificity of long noncoding RNA expression. Biochimica et Biophysica Acta, 1859(1):16-22. DOI 10.1016/j.bbagrm.2015.08.005.

Hu X, Luo J, Lai H, Li M, Zheng X, Nie T, Li F, Li H. 2018. Knockdown of Trnaulap inhibits the proliferation and migration of NIH3T3, JEG-3 and Bewo cells via the PI3K/Akt signaling pathway. Biochem Biophys Res Commun May 11. pii: S0006-291X (18)31120-3. DOI 10.1016/j.bbrc.2018.05.065.

Huang DW, Sherman BT, Lempicki RA. 2009. Bioinformatics enrichment tools: paths toward the comprehensive functional analysis of large gene lists. Nucleic Acids Res 37(1):1-13. DOI 10.1093/nar/gkn923.

Huang DW, Sherman BT, Lempicki RA. 2009. Systematic and integrative analysis of large gene lists using DAVID Bioinformatics Resources. Nature Protoc 4(1):44-57. DOI 10.1038/nprot.2008.211.

\section{Humphries Matthew P, Sundara Rajan Sreekumar, Droop Alastair, et al.}

PeerJ reviewing PDF | (2019:05:37707:1:2:NEW 28 Jul 2019) 
506

507

508

509

510

511

512

513

514

515

516

517

518

519

520

521

522

523

524

525

526

527

528

529

530

531

532

533

534

535

536

537

538

539

540

541

Humphries MP, Sundara Rajan S, Droop A, Suleman CAB, Carbone C, Nilsson C, Honarpisheh H, Cserni G, Dent J, Fulford L, Jordan LB, Jones JL, Kanthan R, Litwiniuk M, Di Benedetto A, Mottolese M, Provenzano E, Shousha S, Stephens M, Walker RA, Kulka J, Ellis IO, Jeffery M, Thygesen HH, Cappelletti V, Daidone MG, Hedenfalk IA, Fjällskog ML, Melisi D, Stead LF, Shaaban AM, Speirs V. 2017. A Case-Matched Gender Comparison Transcriptomic Screen Identifies eIF4E and eIF5 as Potential Prognostic Markers in Male Breast Cancer. Clinical cancer research: an official journal of the American Association for Cancer Research, 23(10). DOI 10.1158/1078-0432.CCR-16-1952.

Hapkova I, Skarda J, Rouleau C, Thys A, Notarnicola C, Janikova M, Bernex F, Rypka M, Vanderwinden JM, Faure S, Vesely J, de Santa Barbara P. 2013. High expression of the RNA-binding protein RBPMS2 in gastrointestinal stromal tumors. Experimental and Molecular Pathology, 94(2). DOI 10.1016/j.yexmp.2012.12.004.

Ishizuka A, Hasegawa Y, Ishida K, Yanaka K, Nakagawa S. 2014. Formation of nuclear bodies by the lncRNA Gomafu-associating proteins Celf3 and SF1. Genes to Cells, 19(9):704-721. DOI 10.1111/gtc.12169.

Kim BK, Yoo HI, Choi K, Lee AR, Yoon SK. 2016. Regulation of Srpr Expression by miR-330-5p Controls Proliferation of Mouse Epidermal Keratinocyte. PLoS One Oct 21, 11(10):e0164896. DOI 10.1371/journal.pone.0164896.

Kim, M.Y., J. Hur, and S. Jeong. 2009. Emerging roles of RNA and RNA-binding protein network in cancer cells. BMB Rep 42(3): p. 125-30. DOI 10.5483/BMBRep.2009.42.3.125.

Lambert SA, Jolma A, Campitelli LF, Das PK, Yin Y, Albu M, Chen X, Taipale J, Hughes TR, Weirauch MT. 2018. The Human Transcription Factors. Cell. 172(4):650-665. DOI: 10.1016/j.cell.2018.01.029.

Leah L. Zagore, Sarah E. Grabinski, Thomas J. 2015. Sweet, Molly M. Hannigan, R. Michael Sramkoski, Qin Li, Donny D. Licatalosi. RNA Binding Protein Ptbp2 Is Essential for Male Germ Cell Development. Mol Cell Biol Dec, 35(23): 4030-4042. DOI 10.1128/MCB.00676-15.

Li Jun-Hao, Liu Shun, Zhou Hui, Qu Liang-Hu, Yang Jian-Hua. 2013. starBase v2.0: decoding miRNAceRNA, miRNA-ncRNA and protein-RNA interaction networks from large-scale CLIP-Seq data. Nucleic Acids Res. 42: D92-7. DOI 10.1093/nar/gkt1248.

Liu H, Liang S, Yang X, Ji Z, Zhao W, Ye X, Rui J. 2015. RNAi-mediated RPL34 knockdown suppresses the growth of human gastric cancer cells. Oncol Rep Nov, 34(5):2267-72. DOI 10.3892/or.2015.4219.

Pavlyukov MS, Yu H, Bastola S, Minata M, Shender VO, Lee Y, Zhang S, Wang J, Komarova S, Wang J, Yamaguchi S, Alsheikh HA, Shi J, Chen D, Mohyeldin A, Kim SH, Shin YJ, Anufrieva K, Evtushenko EG, Antipova NV, Arapidi GP, Govorun V, Pestov NB, Shakhparonov MI, Lee LJ, Nam DH, Nakano I. 2018. Apoptotic Cell-Derived Extracellular Vesicles Promote Malignancy of Glioblastoma Via Intercellular Transfer of Splicing Factors. Cancer Cell, 34(1). DOI 10.1016/j.ccell.2018.05.012.

Minoru Kato, Min Wei, Shotaro Yamano, Anna Kakehashi, Satoshi Tamada, Tatsuya Nakatani, Hideki Wanibuchi. 2012. DDX 39 acts as a suppressor of invasion for bladder cancer. Cancer Science, 103(7). DOI 10.1111/j.1349-7006.2012.02298.x.

Peer] reviewing PDF | (2019:05:37707:1:2:NEW 28 Jul 2019) 
542 Molly M. Hannigan, Leah L, Zagore, Donny D, Licatalosi. 2017. Ptbp2 controls an alternative splicing network

543

544

545

546

547

548

549

550

551

552

553

554

555

556

557

558

559

560

561

562

563

564

565

566

567

568

569

570

571

572

573

574

575

576

577

required for cell communication during spermatogenesis. Cell Rep, Jun 20, 19(12):2598-2612. DOI 10.1016/j.celrep.2017.05.089.

Moore, M.J., and Proudfoot, N.J. 2009. Pre-mRNA processing reaches back to transcription and ahead to translation. Cell 136, 688-700. DOI 10.1016/j.cell.2009.02.001.

Ogawa Y, Kakumoto K, Yoshida T, Kuwako KI, Miyazaki T, Yamaguchi J, Konno A, Hata J, Uchiyama Y, Hirai H, Watanabe M, Darnell RB, Okano H, Okano HJ. 2018. Elavl3 is essential for the maintenance of Purkinje neuron axons. Sci Rep Feb 9, 8(1):2722. DOI 10.1038/s41598-018-21130-5.

Otsuka K, Yamamoto Y, Ochiya T. 2018. Regulatory role of resveratrol, a microRNA-controlling compound, in HNRNPA1 expression, which is associated with poor prognosis in breast cancer. Oncotarget 15, 9(37) :2471824730. DOI 10.18632/oncotarget.25339.

Peter Langfelder and Steve Horvath. 2008. WGCNA: an R package for weighted correlation network analysis. BMC Bioinformatics 9:559. DOI 10.1186/1471-2105-9-559.

Piao J, Chen L, Jin T, Xu M, Quan C, Lin Z. 2018. Paip1 affects breast cancer cell growth and represents a novel prognostic biomarker. Hum Pathol Mar, 73:33-40. DOI 10.1016/j.humpath.2017.10.037.

Qin Li, Sika Zheng, Areum Han, Chia-Ho Lin, Peter Stoilov, Xiang-Dong Fu, Douglas L Black. 2014. The splicing regulator PTBP2 controls a program of embryonic splicing required for neuronal maturation. eLife 3: e01201. DOI 10.7554/eLife.01201.

Robinson, M., D. Mccarthy, and G.K. Smyth. 2010. edgeR: differential expression analysis of digital gene expression data. Journal of Hospice \& Palliative Nursing 4(4): p. 206-207. DOI 10.1093/bioinformatics/btp616.

Saijo Saki, Kuwano Yuki, Masuda Kiyoshi, Nishikawa Tatsuya, Rokutan Kazuhito, Nishida Kensei. 2016. Serine/arginine-rich splicing factor 7 regulates p21-dependent growth arrest in colon cancer cells. The Journal of Medical Investigation, 63(3.4):219-226. DOI 10.2152/jmi.63.219.

Shi R, Yu X, Wang Y, Sun J, Sun Q, Xia W. 2017. Expression profile, clinical significance, and biological function of insulin-like growth factor 2 messenger RNA-binding proteins in non-small cell lung cancer. Tumor Biology, 39(4):101042831769592. DOI 10.1177/1010428317695928.

Shuaibin Lian, Tianliang Liu, Shengli Jing, Hongyu Yuan, Zaibao Zhang, and Lin Cheng. 2018. Intrachromosomal colocalization strengthens co-expression, co-modification and evolutionary conservation of neighboring genes. BMC Genomics, 19(1):455-. DOI 10.1186/s12864-018-4844-1.

Sobin L H, Gospodarowicz M K, Wittekind C. 1992. TNM Classification of Malignant Tumours, 7th Edition// TNM classification of malignant tumours. Springer-Verlag. DOI 10.1057/9780230271357_47.

Soyal S M, Zara G, Ferger B, Felder TK, Kwik M, Nofziger C, Dossena S, Schwienbacher C, Hicks AA, Pramstaller PP, Paulmichl M, Weis S, Patsch W. 2019. The PPARGC1A locus and CNS-specific PGC-1 $\alpha$ isoforms are associated with Parkinson's Disease Neurobiology of Disease, 121:34-46. DOI 10.1016/j.nbd.2018.09.016.

PeerJ reviewing PDF | (2019:05:37707:1:2:NEW 28 Jul 2019) 
578

579

580

581

582

583

584

585

586

587

588

589

590

591

592

593

594

595

596

597

598

599

600

601

602

603

604

605

606

607

608

609

610

611

612

Stefanie Gerstberger, Markus Hafner and Thomas Tuschl. 2014. A census of human RNA-binding Proteins. Nature Reviews Genetics 15: 829-845. DOI 10.1038/nrg3813.

St. Laurent G, Wahlestedt C, Kapranov P. 2015. The Landscape of long noncoding RNA classification. Trends in Genetics, 31(5):239-251. DOI 10.1016/j.tig.2015.03.007.

Cao TT, Lin SH, Fu L, Tang Z, Che CM, Zhang LY, Ming XY, Liu TF, Tang XM, Tan BB, Xiang D, Li F, Chan OY, Xie D, Cai Z, Guan XY. 2017. Eukaryotic translation initiation factor 5A2 promotes metabolic reprogramming in hepatocellular carcinoma cells. Carcinogenesis, 38(1). DOI 10.1093/carcin/bgw1 19.

Vergara IA, Erho N, Triche TJ, Ghadessi M, Crisan A, Sierocinski T, Black PC, Buerki C, Davicioni E. 2012. Genomic "Dark Matter" in Prostate Cancer: Exploring the Clinical Utility of ncRNA as Biomarkers. Frontiers in Genetics, 3. DOI 10.3389/fgene.2012.00023.

Wang T, Ma S, Qi X, Tang X, Cui D, Wang Z, Chi J, Li P, Zhai B. 2016. Long noncoding RNA ZNFX1-AS1 suppresses growth of hepatocellular carcinoma cells by regulating the methylation of miR-9. Onco Targets Ther Aug 12:9:5005-14. DOI 10.2147/OTT.S103329.

Wurth, Laurence. 2012. Versatility of RNA-Binding Proteins in Cancer. Comparative and Functional Genomics, 2012:1-11. DOI 10.1155/2012/178525.

Xiao L, Lanz RB, Frolov A, Castro PD, Zhang Z, Dong B, Xue W, Jung SY, Lydon JP, Edwards DP, Mancini MA, Feng Q, Ittmann MM, He B. 2016. The Germ Cell Gene TDRD1 as an ERG Target Gene and a Novel Prostate Cancer Biomarker. Prostate Oct, 76(14):1271-84. DOI 10.1002/pros.23213.

Yamauchi T, Masuda T, Canver MC, Seiler M, Semba Y, Shboul M, Al-Raqad M, Maeda M, Schoonenberg VAC, Cole MA, Macias-Trevino C, Ishikawa Y, Yao Q, Nakano M, Arai F, Orkin SH, Reversade B, Buonamici S, Pinello L, Akashi K, Bauer DE, Maeda T. 2018. Genome-wide CRISPR-Cas9 Screen Identifies Leukemia-Specific Dependence on a Pre-mRNA Metabolic Pathway Regulated by DCPS. Cancer Cell Mar 12, 33(3):386-400.e5. DOI 10.1016/j.ccell.2018.01.012.

Yang S, Cui J, Yang Y, Liu Z, Yan H, Tang C, Wang H, Qin H, Li X, Li J, Wang W, Huang Y, Gao H. 2016. Over-expressed RPL34 promotes malignant proliferation of non-small cell lung cancer cells. Gene Jan 15, 576(1):421-8. DOI 10.1016/j.gene.2015.10.053.

Yang Y, Quan L, Ling Y. 2018. RBMS3 Inhibits the Proliferation and Metastasis of Breast Cancer Cells. Oncol Res Jan 19, 26(1):9-15. DOI 10.3727/096504017X14871200709504.

Yin Z H, Jiang X W, Shi W B, Gui Q L, Yu D F. 2017. Expression and Clinical Significance of ILF2 in Gastric Cancer. Disease markers, 2017(1):1-9. DOI 10.1155/2017/4387081.

Yuan J H, Liu X N, Wang T T, Pan W, Tao Q F, Zhou W P. 2017. The MBNL3 splicing factor promotes hepatocellular carcinoma by increasing PXN expression through the alternative splicing of IncRNA-PXN-AS1. Nature Cell Biology. DOI 10.1038/ncb3538.

Zhang X, Shen B, Cui Y. 2019. Ago HITS-CLIP expands microRNA-mRNA interactions in nucleus and cytoplasm of gastric cancer cells. BMC Cancer, 19(1). DOI 10.1186/s12885-018-5246-0.

Peer) reviewing PDF | (2019:05:37707:1:2:NEW 28 Jul 2019) 
613 ZeLin Wang, Bin Li, YuXia Luo, Qiao Lin, ShuRong Liu, XiaoQin Zhang, Hui Zhou, JianHua Yang, and

614

615

616

617

618

619

620

621

622

623

624

625

626

627

628

629

630

631

632

633

634

635

636

637

638

639

640

641

642

643

644

645

646

647

648

649

650

LiangHu Qu. 2018. Comprehensive Genomic Characterization of RNA-Binding Proteins across Human Cancers. Cell Reports 22, January 2, 286-298. DOI 10.1016/j.celrep.2017.12.035.

\section{Zhou Du, Teng Fei, Roel G W Verhaak, Zhen Su, Yong Zhang, Myles Brown, Yiwen Chen, X Shirley Liu}

2013. Integrative genomic analyses reveal clinically relevant long noncoding RNAs in human cancer. Nature Structural \& Molecular Biology, 20(7):908-913. DOI 10.1038/nsmb.2591.

\section{Figures}

Fig. 1. Workflow chart showing the different steps presented in this study. The flow chart shows the acquisition and preparation of data (Pink box), differentially expressed genes analysis and module detection (Light yellow box), networks construction and function analysis (Pale green box). RBP, RNA-binding protein; TCGA, the Cancer Genome Atlas; TF, transcription factor; lncRNA, long non-coding RNA.

Fig. 2. The different expression patterns of RBPs and non-RBPs in cancers and normal tissues. (A) The standard expression deviation of RBPs in 20 types of cancers and normal tissues. (B) The standard expression deviation of non-RBPs in 20 types of cancers and normal tissues. (C) The relative expression deviation of RBPs and non-RBPs. (D) The standard expression deviation of up- regulated RBPs in cancers and normal tissues. (E) The standard expression deviation of down- regulated RBPs in cancers and normal tissues. (F) The expression FPKM values of up- regulated RBPs in cancers and normal tissues. (G) The expression FPKM values of down- regulated RBPs in cancers and normal tissues. Significance values calculated from the Mann-Whitney $U$ test are shown.

Fig. 3. An expression heat map of 801 differentially expressed RBPs shared by 16 cancer types in six systems. (A) Respiratory system. (B)Liver and gall. (C)Nervous system. (D) Alimentary system. (E) Reproductive system. (F) Urinary system. Red and blue represent high and low expression. The right column of each system is the Pearson coefficient $R$ of the corresponding cluster. Gene cluster with $\mathrm{R}>0.5$ was marked with dark yellow, which represents the similar expression pattern.

Fig. 4. The co-expression modules detection of $\mathrm{CHOL}$ and $\mathrm{KICH}$, respectively. (A) and (B) Co-expression modules of $\mathrm{CHOL}$ and KICH correlated with their clinical TNM stage information, respectively. Different color represents the gene modules with different expression pattern. The first, middle, and last column are detected gene modules related to $M, N$, and $T$ stages, respectively. The two numbers in each module " $a(b)$ " represent coefficients of co-expression and statistical significance, $a$ is the co-expression coefficient and $b$ is the corresponding $P$-value. The modules with coefficient larger than 0.5 and $P$-value smaller than 0.05 were considered as the related modules. (C) and (D) Co-expression networks of RBPs and non-RBPs in gene modules (green, red and royal blue) related with $M$ - and $N$-stages for $\mathrm{CHOL}$ and $\mathrm{KICH}$, respectively. Inner circle are key RBPs, outer circle are the non-RBPs. Triangular represents the up-regulated genes, block represents the down-regulated genes.

Fig. 5. The co-expression networks of key RBPs and lncRNAs. (A) The co-expression network of two key RBPs related to $M$ stage (C2orf15 and DCPS) and lncRNAs for CHOL. (B) The co-expression network of three key RBPs (TDRD1, TDRD9 and $C E L F 4)$ related to $M$ stage and lncRNAs for KICH. Red and green represent up- and down-regulated DE genes.

Peer] reviewing PDF | (2019:05:37707:1:2:NEW 28 Jul 2019) 
651

652

653

654

655

656

657

658

659 Tables

660

661
Fig. 6. The co-expression networks of DE RBPs and TFs. (A) and (C) The co-expression networks of DE RBPs and TFs for CHOL and KICH respectively. (B) and (D) The corresponding pie chart of TFs co-expressed with DE RBPs for CHOL and $\mathrm{KICH}$, respectively. Inner circle are key TFs, outer circle are DE RBPs. Red and green in outer circle represent up- and downregulated RBPs. Block represents the key RBPs identified by module detection, circle dot represents other DE RBPs.

Table 1: Differentially expressed RBPs and non-RBPs across 20 types of cancers in seven systems. 


\section{Figure 1}

Workflow chart showing the different steps presented in this study.

The flow chart shows the acquisition and preparation of data (Pink box), differentially expressed genes analysis and module detection (Light yellow box), networks construction and function analysis (Pale green box). RBP, RNA-binding protein; TCGA, the Cancer Genome Atlas; TF, transcription factor; IncRNA, long non-coding RNA.

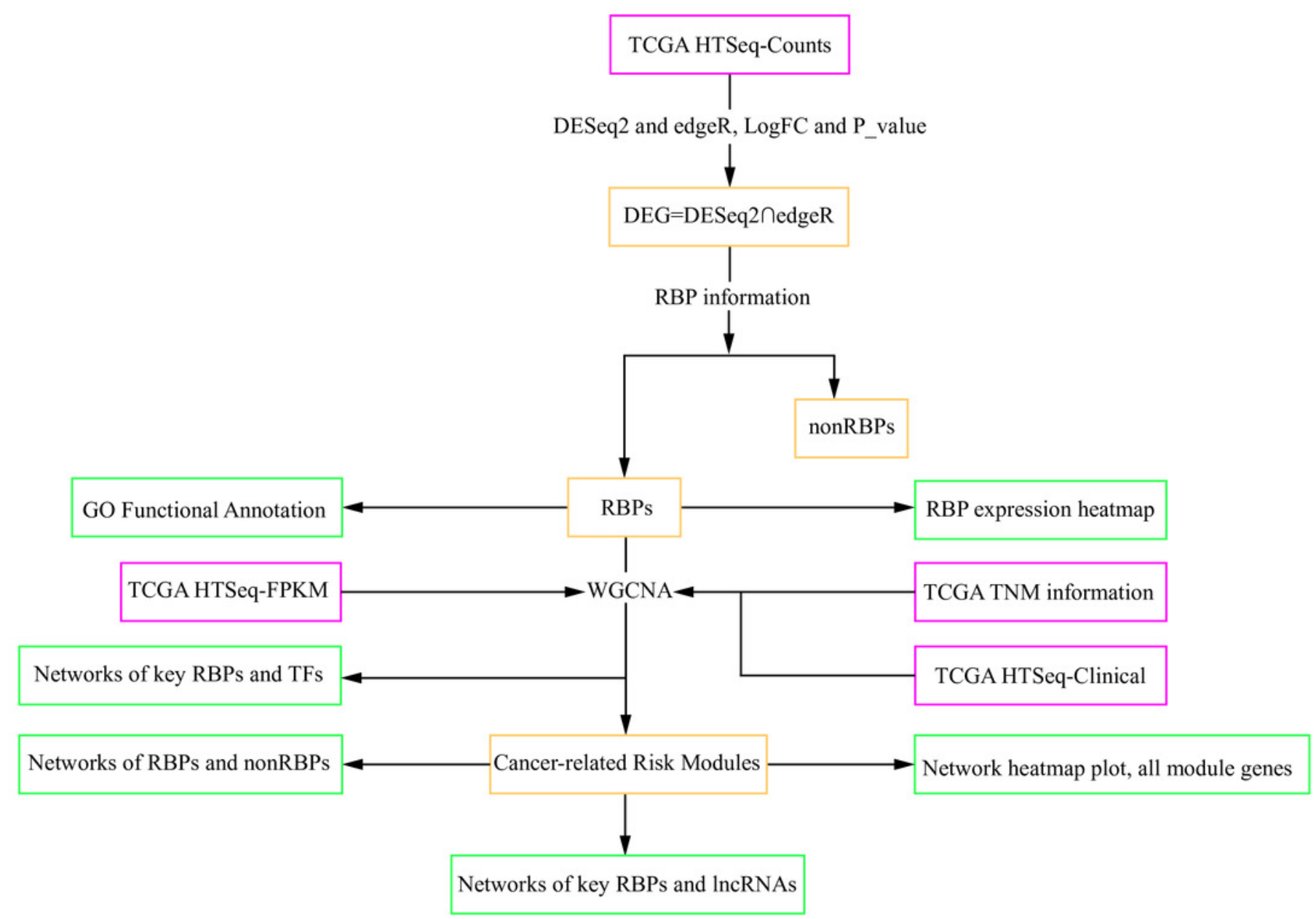




\section{Figure 2}

The different expression patterns of RBPs and non-RBPs in cancers and normal tissues.

(A) The standard expression deviation of RBPs in 20 types of cancers and normal tissues. (B) The standard expression deviation of non-RBPs in 20 types of cancers and normal tissues. (C) The relative expression deviation of RBPs and non-RBPs. (D) The standard expression deviation of up- regulated RBPs in cancers and normal tissues. (E) The standard expression deviation of down- regulated RBPs in cancers and normal tissues. (F) The expression FPKM values of up- regulated RBPs in cancers and normal tissues. (G) The expression FPKM values of down- regulated RBPs in cancers and normal tissues. Significance values calculated from the Mann-Whitney $U$ test are shown. 

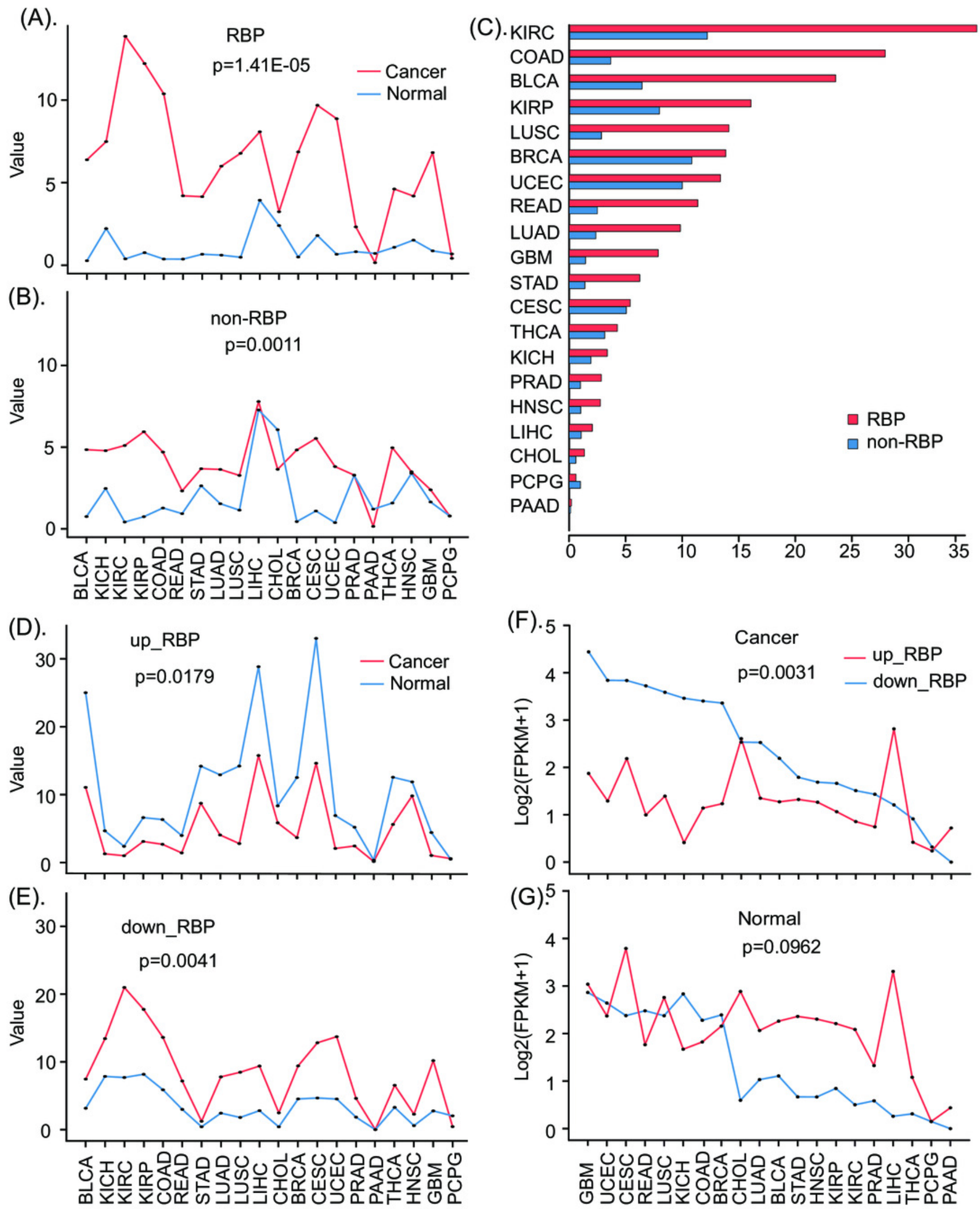
Figure 3

An expression heat map of 801 differentially expressed RBPs shared by 16 cancer types in six systems.

(A) Respiratory system. (B)Liver and gall. (C)Nervous system. (D) Alimentary system. (E)

Reproductive system. (F) Urinary system. Red and blue represent high and low expression. The right column of each system is the Pearson coefficient $R$ of the corresponding cluster. Gene cluster with $\mathrm{R}>0.5$ was marked with dark yellow, which represents the similar expression pattern.

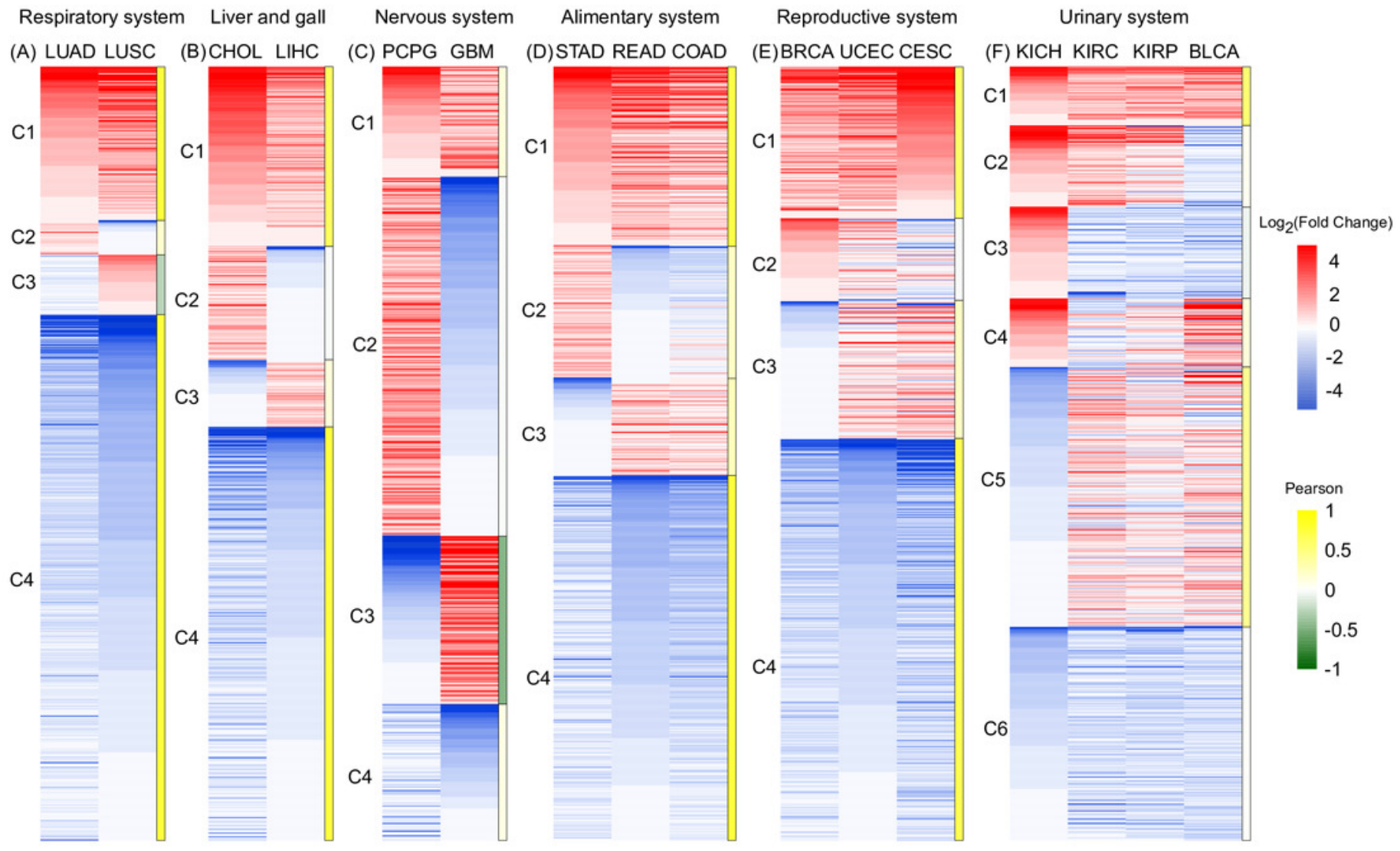


PeEHOL

Module-trait relationships

\begin{tabular}{|c|c|c|c|}
\hline greenoyellow & $0.18(0.4)$ & $-0.045(0.8)$ & $-0.13(0.5)$ \\
\hline midnightblue & $-0.12(0.6)$ & $-0.0068(1)$ & $0.058(0.8)$ \\
\hline green & $0.39(0.05)$ & $0.47(0.01)$ & $0.63(5 e-04)$ \\
\hline purple & $0.04(0.8)$ & $0.18(0.4)$ & $0.15(0.5)$ \\
\hline red & $0.22(0.3)$ & $0.53(0.005)$ & $0.61(0.001)$ \\
\hline royalblue & $0.45(0.02)$ & $0.52(0.007)$ & $0.1(0.6)$ \\
\hline darkred & $0.23(0.2)$ & $0.42(0.03)$ & $-0.059(0.8)$ \\
\hline lightgreen & $0.2(0.3)$ & 0.007 (1) & $0.11(0.6)$ \\
\hline cyan & $0.49(0.01)$ & $0.058(0.8)$ & $0.014(0.9)$ \\
\hline grey60 & $0.22(0.3)$ & $-0.0056(1)$ & $-0.014(0.9)$ \\
\hline grey & $0.33(0.1)$ & $0.45(0.02)$ & $0.32(0.1)$ \\
\hline
\end{tabular}

$\mathrm{N}$

$\mathrm{M}$

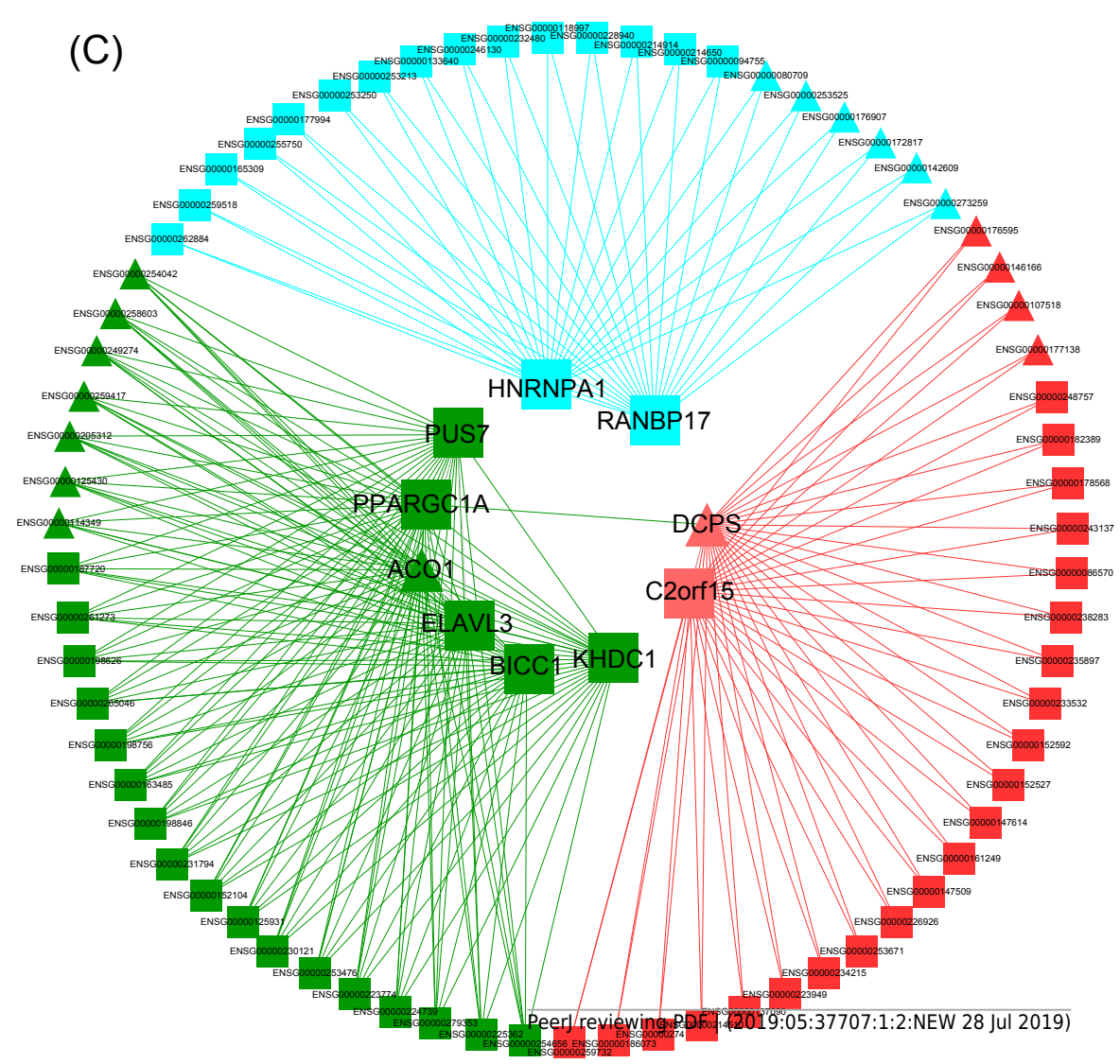

Module-trait relationships

\begin{tabular}{|} 
orange \\
brown \\
black \\
green \\
cyan \\
red \\
saddlebrown \\
royalblue \\
paleturquoise \\
salmon \\
blue
\end{tabular}

\begin{tabular}{|l|c|c|}
\hline$-0.22(0.3)$ & $-0.23(0.2)$ & $-0.077(0.7)$ \\
$0.22(0.3)$ & $-0.05(0.8)$ & $-0.056(0.8)$ \\
$0.24(0.2)$ & $-0.056(0.8)$ & $-0.0098(1)$ \\
$0.3(0.1)$ & $0.69(6 \mathrm{e}-05)$ & $-0.038(0.8)$ \\
$0.27(0.2)$ & $0.025(0.9)$ & $-0.041(0.8)$ \\
$0.27(0.2)$ & $0.7(4 \mathrm{e}-05)$ & $-0.028(0.9)$ \\
$0.12(0.5)$ & $0.31(0.1)$ & $-0.025(0.9)$ \\
$0.26(0.2)$ & $0.33(0.09)$ & $0.96(4 \mathrm{e}-15)$ \\
$-0.23(0.2)$ & $-0.017(0.9)$ & $0.0055(1)$ \\
$0.27(0.2)$ & $-0.022(0.9)$ & $0.027(0.9)$ \\
$0.23(0.2)$ & $-0.091(0.7)$ & $-0.087(0.7)$ \\
\hline
\end{tabular}

M

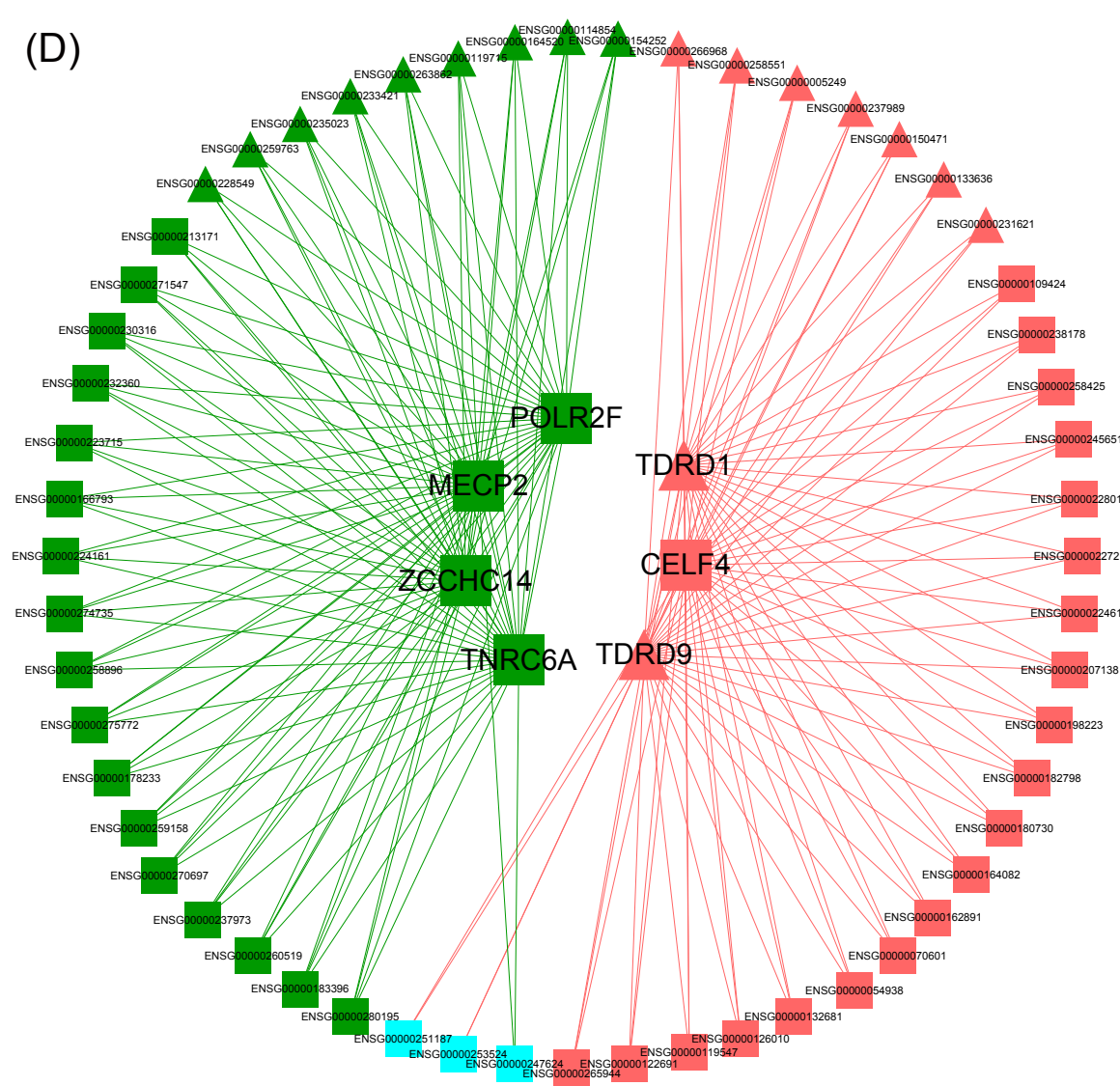


(A) $\mathrm{CHOL}$

Manuscript to be revie(B) $\mathrm{KICH}$

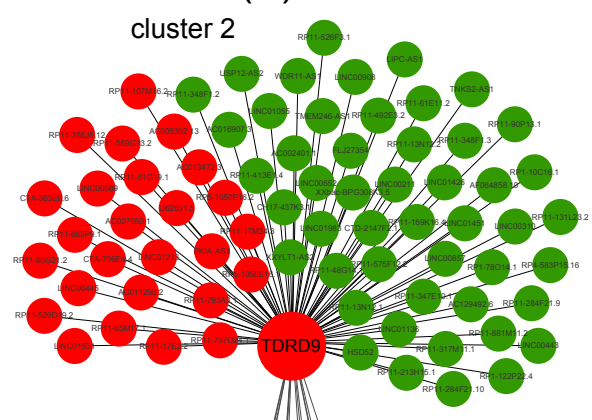

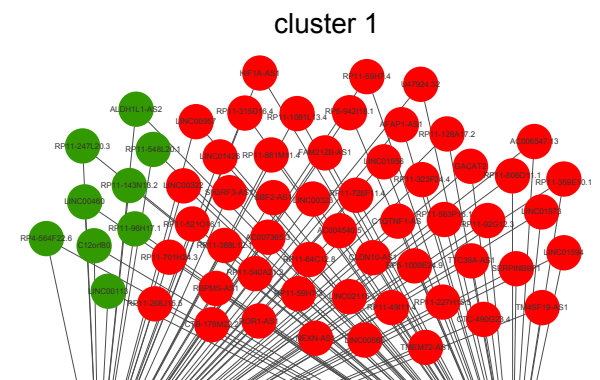

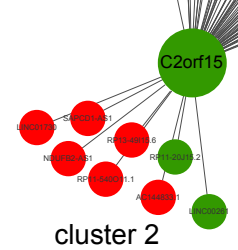

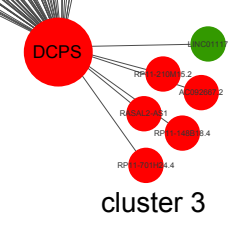

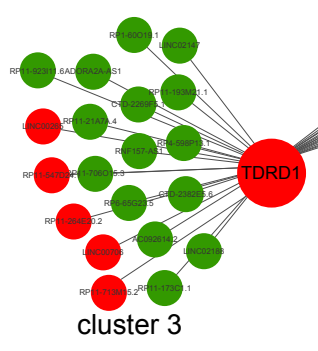

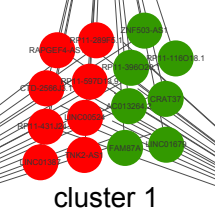

-

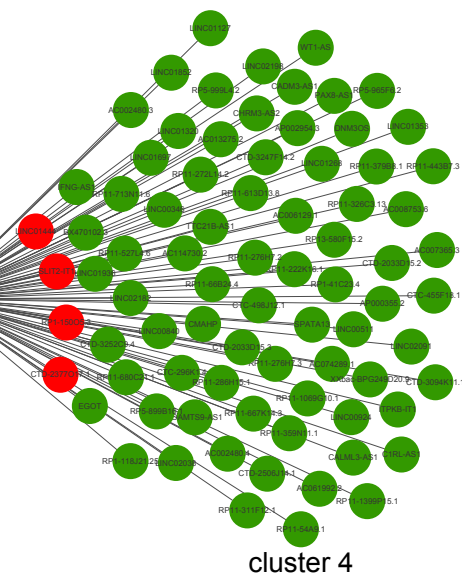




\section{(A) $\mathrm{CHOL}-\mathrm{TFs}$}

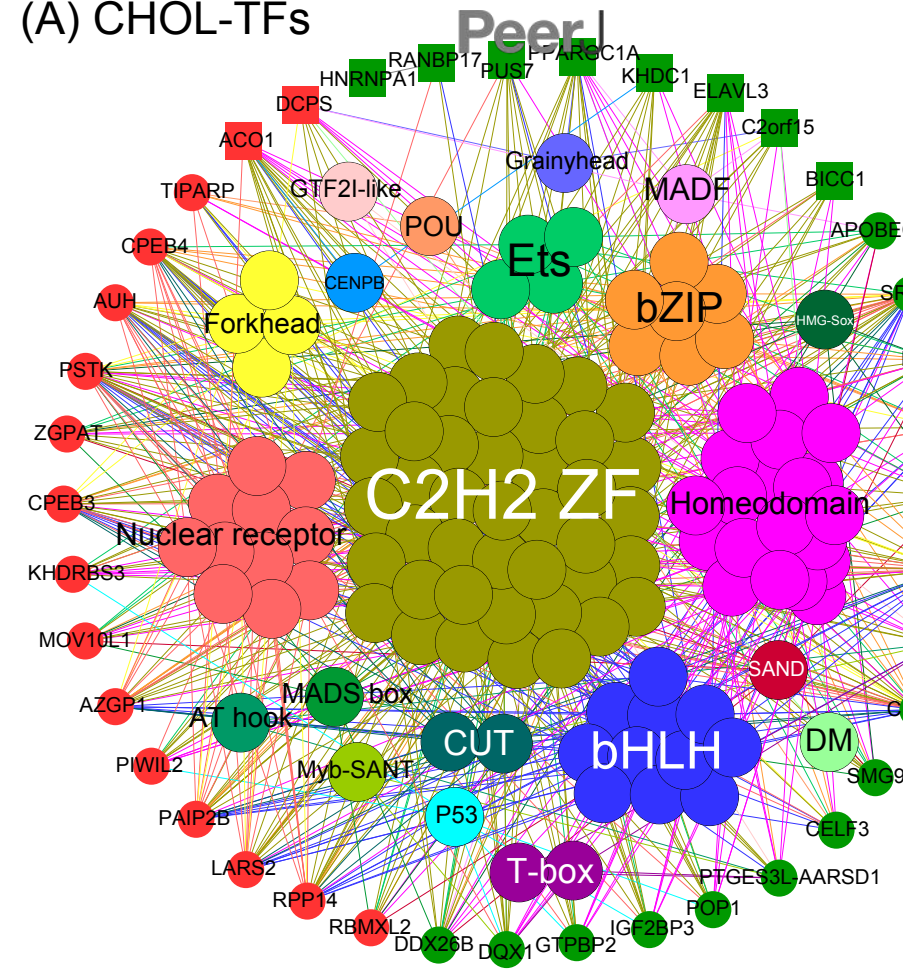

(C) $\mathrm{KICH}-\mathrm{TFs}$

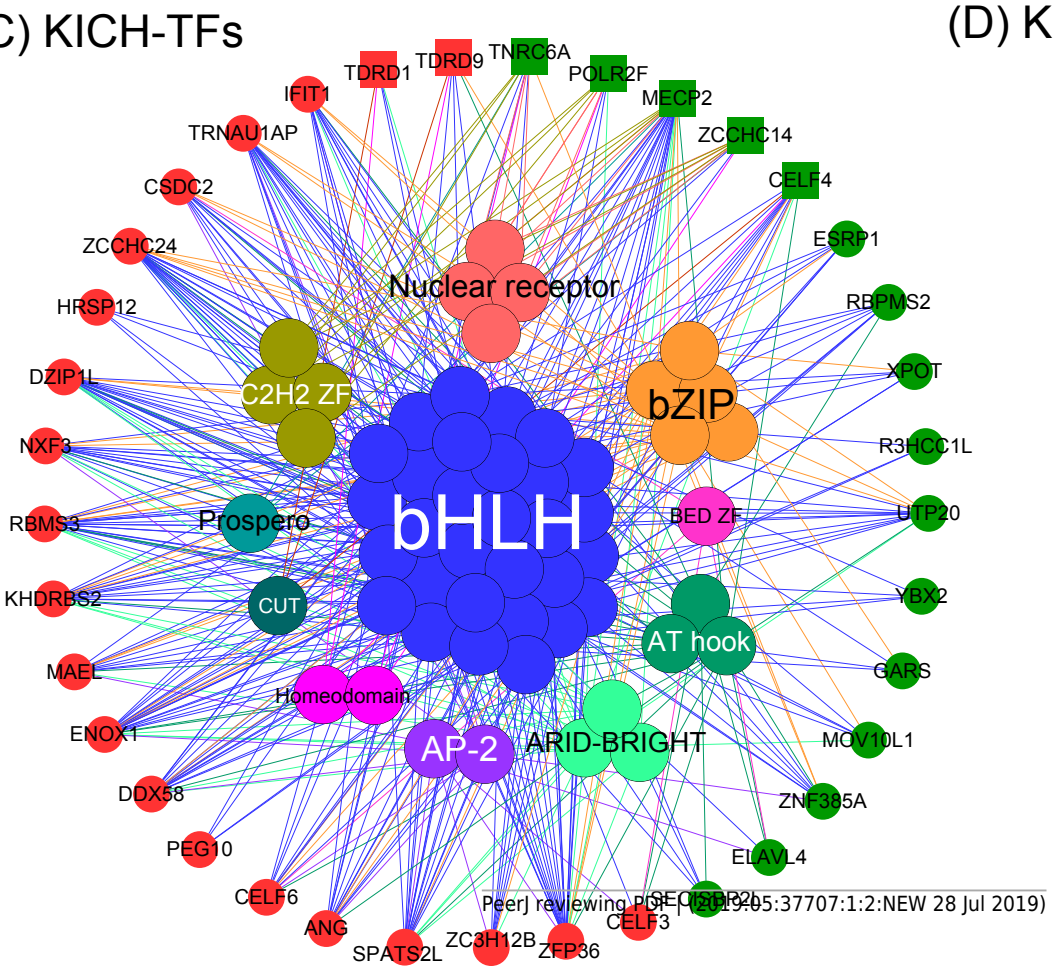

(B) CHOL-Pieqcharts cript to be baipiewed

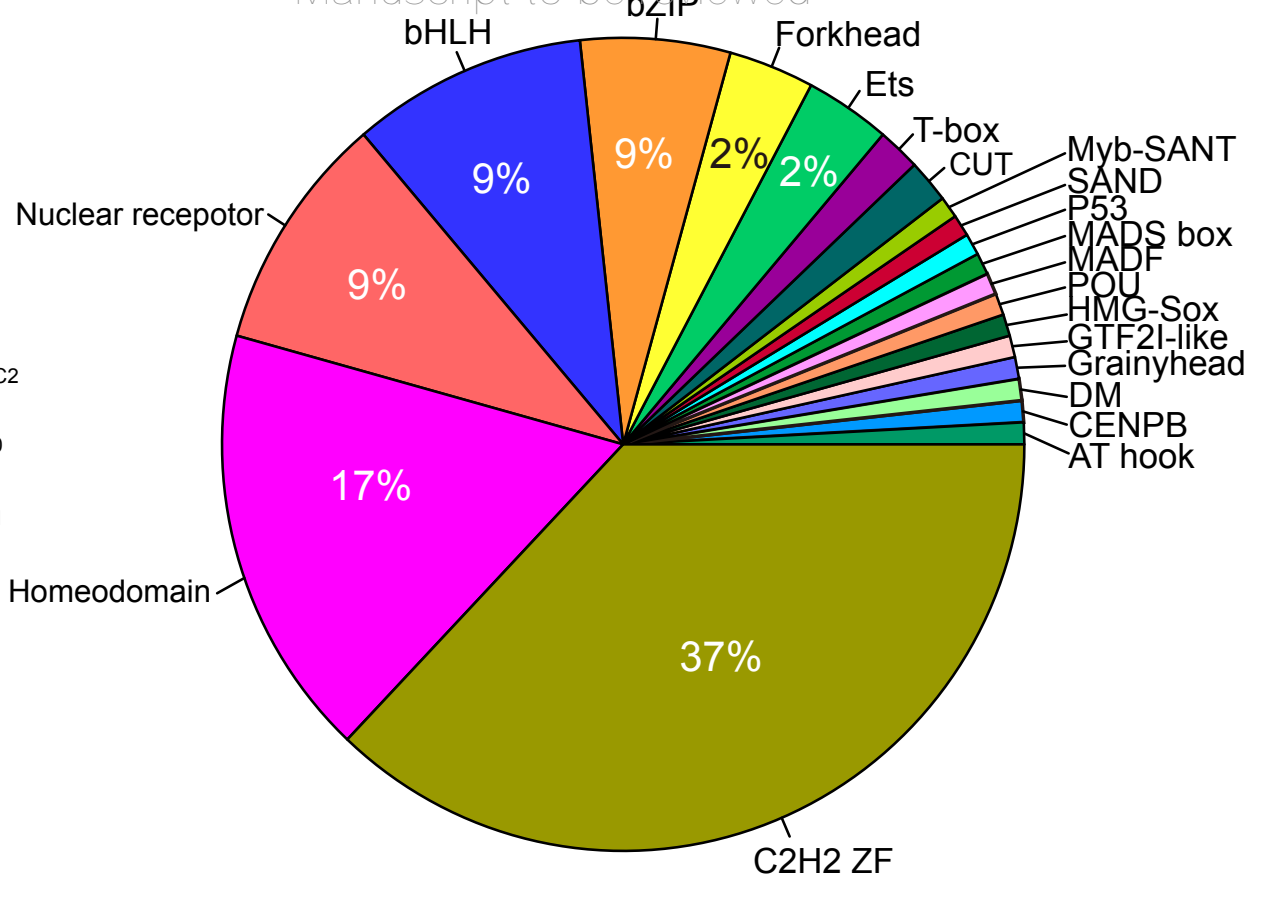

(D) $\mathrm{KICH}$-Pie chart

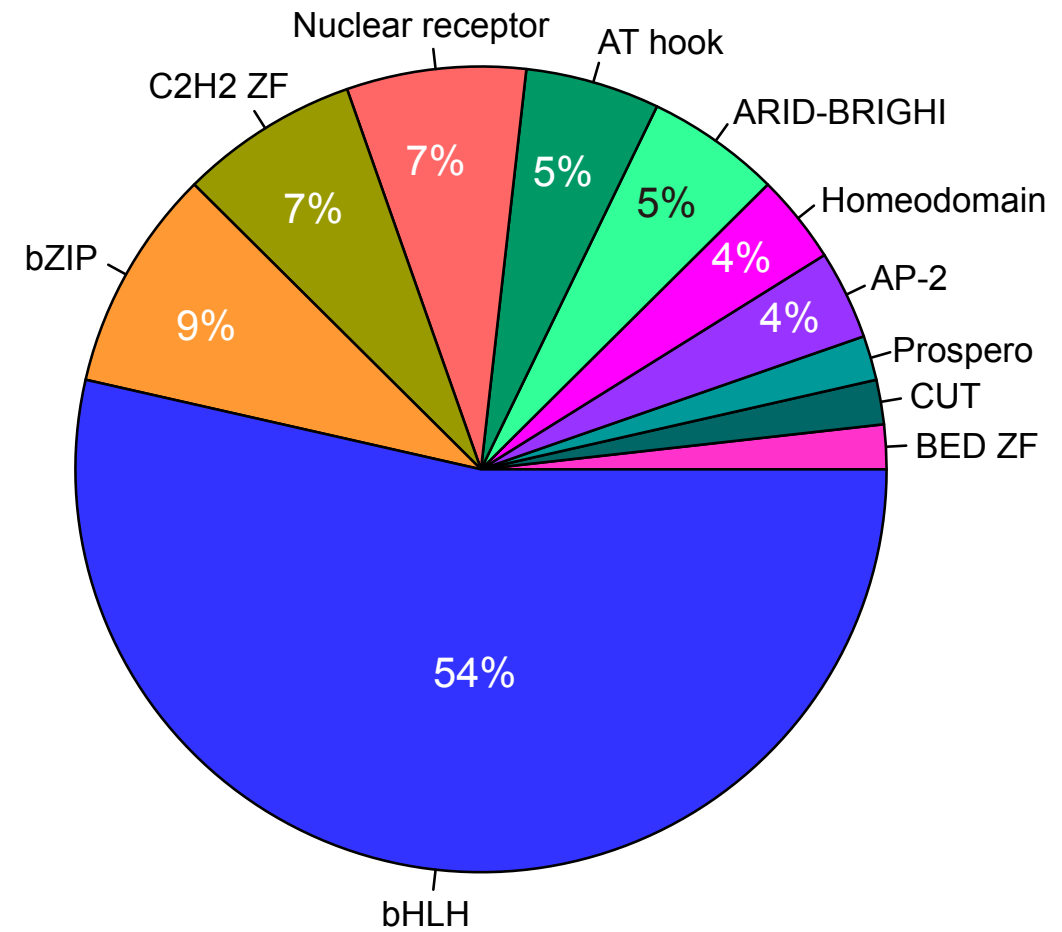




\section{Table 1 (on next page)}

Differentially expressed RBPs and non-RBPs across 20 types of cancers in seven systems. 


\begin{tabular}{|c|c|c|c|c|c|c|c|}
\hline System & Cancer & Cancer type & $\begin{array}{l}\text { Total } \\
\text { DEG } \\
\text { genes }\end{array}$ & $\begin{array}{l}\text { Total } \\
\text { non-RBPs }\end{array}$ & $\begin{array}{l}\text { Total } \\
\text { RBPs }\end{array}$ & $\begin{array}{l}\text { Up- } \\
\text { regulated } \\
\text { RBPs }\end{array}$ & $\begin{array}{l}\text { Down- } \\
\text { regulated } \\
\text { RBPs }\end{array}$ \\
\hline \multirow{4}{*}{$\begin{array}{l}\text { landular } \\
\text { system }\end{array}$} & PAAD & Pancreatic adenocarcinoma & 260 & 259 & 1 & 1 & 0 \\
\hline & THCA & Thyroid carcinoma & 4804 & 4765 & 39 & 14 & 25 \\
\hline & PRAD & Prostate adenocarcinoma & 4777 & 4722 & 55 & 34 & 21 \\
\hline & HNSC & $\begin{array}{l}\text { Head and neck squamous cell } \\
\text { carcinoma }\end{array}$ & 7421 & 7335 & 86 & 32 & 54 \\
\hline \multirow{2}{*}{$\begin{array}{l}\text { Respiratory } \\
\text { system }\end{array}$} & LUSC & Lung squamous cell carcinoma & 12235 & 11972 & 263 & 54 & 209 \\
\hline & LUAD & Lung adenocarcinoma & 9004 & 8859 & 145 & 35 & 110 \\
\hline \multirow{3}{*}{$\begin{array}{l}\text { Alimentary } \\
\text { system }\end{array}$} & READ & Rectum adenocarcinoma & 8620 & 8430 & 190 & 60 & 130 \\
\hline & COAD & Colon adenocarcinoma & 8954 & 8773 & 181 & 44 & 137 \\
\hline & STAD & Stomach adenocarcinoma & 9037 & 8932 & 105 & 39 & 66 \\
\hline \multirow[t]{4}{*}{ Urinary system } & $\mathrm{KICH}$ & Kidney chromophobe & 9926 & 9758 & 168 & 76 & 92 \\
\hline & KIRC & Kidney renal clear cell carcinoma & 11478 & 11370 & 108 & 37 & 71 \\
\hline & KIRP & $\begin{array}{l}\text { Kidney renal papillary cell } \\
\text { carcinoma }\end{array}$ & 8293 & 8189 & 104 & 33 & 71 \\
\hline & BLCA & Bladder urothelial carcinoma & 7146 & 7025 & 121 & 53 & 68 \\
\hline \multirow[t]{3}{*}{$\begin{array}{l}\text { Reproductive } \\
\text { system }\end{array}$} & CESC & $\begin{array}{l}\text { Cervical squamous cell carcinoma } \\
\text { and endocervical adenocarcinoma }\end{array}$ & 4302 & 4154 & 148 & 57 & 91 \\
\hline & UCEC & $\begin{array}{l}\text { Uterine corpus endometrial } \\
\text { carcinoma }\end{array}$ & 9046 & 8869 & 177 & 64 & 113 \\
\hline & BRCA & Breast invasive carcinoma & 7426 & 7298 & 128 & 36 & 92 \\
\hline \multirow{2}{*}{$\begin{array}{l}\text { Nervous } \\
\text { system }\end{array}$} & GBM & Glioblastoma multiforme & 11946 & 11628 & 318 & 118 & 200 \\
\hline & PCPG & $\begin{array}{l}\text { Pheochromocytoma and } \\
\text { paraganglioma }\end{array}$ & 4717 & 4560 & 157 & 101 & 56 \\
\hline \multirow{2}{*}{$\begin{array}{l}\text { Liver and } \\
\text { gall system }\end{array}$} & LIHC & Liver hepatocellular carcinoma & 6677 & 6576 & 101 & 25 & 76 \\
\hline & CHOL & Cholangiocarcinoma & 10219 & 10030 & 189 & 80 & 109 \\
\hline
\end{tabular}

